Review

\title{
Concurrence Measurement for the Two-Qubit Optical and Atomic States
}

\section{Lan Zhou ${ }^{1,2}$ and Yu-Bo Sheng ${ }^{2,3, *}$}

${ }^{1}$ College of Mathematics \& Physics, Nanjing University of Posts and Telecommunications, Nanjing 210003, China; E-Mail: zhoul@njupt.edu.cn

${ }^{2}$ Key Lab of Broadband Wireless Communication and Sensor Network Technology, Nanjing University of Posts and Telecommunications, Ministry of Education, Nanjing 210003, China

${ }^{3}$ Institute of Signal Processing Transmission, Nanjing University of Posts and Telecommunications, Nanjing 210003, China

* Author to whom correspondence should be addressed; E-Mail: shengyb@njupt.edu.cn;

Tel./Fax: +86-025-83492417.

Academic Editor: Jay Lawrence

Received: 27 March 2015 / Accepted: 16 June 2015 / Published: 19 June 2015

\begin{abstract}
Concurrence provides us an effective approach to quantify entanglement, which is quite important in quantum information processing applications. In the paper, we mainly review some direct concurrence measurement protocols of the two-qubit optical or atomic system. We first introduce the concept of concurrence for a two-qubit system. Second, we explain the approaches of the concurrence measurement in both a linear and a nonlinear optical system. Third, we introduce some protocols for measuring the concurrence of the atomic entanglement system.
\end{abstract}

Keywords: quantum entanglement; concurrence; quantum nondemolition measurement

\section{Introduction}

Entanglement has been regarded as a key source in quantum information processing (QIP) since it was first introduced by Einstein, Podolsky and Rosen [1] and Schrödinger [2]. Nowadays, entanglement is required in nearly all quantum communication and computation protocols [3], such as quantum teleportation [4-7], dense coding [8,9], quantum key distribution (QKD) [10-12], quantum secure 
direct communication [13-15], quantum computation [16-19], and other protocols [20-23]. During the past decade, with the rapid development of the experimental process on quantum control, there is a rapidly growing interest in entanglement generation [24-32]. For example, in 2005, six or eight ions were entangled [24,25]. In 2007, photons were used to demonstrate entanglement between six or ten qubits [26,27]. Actually, at an earlier time, with the help of spin squeezing, entanglement of $10^{7}$ atoms was created in cold atomic clouds [28], and large-scale entangling operations were realized in optical lattices of $10^{5}$ two-state atoms [29].

How to quantify the entanglement is important in QIP applications. Bell inequalities [33] and entanglement witnesses [34-36] are the early approaches to realize the entanglement characterization. However, it is difficult for them to provide satisfactory results of the entanglement measurement, for they only disclose the entanglement of some states while failing for other states [37]. Another indirect entanglement measurement method is the quantum state tomographic reconstruction [38-40]. In quantum state tomographic reconstruction, we need to first reconstruct the density matrix of an unknown quantum state by measuring a complete set of observables and then calculate its entanglement. However, this approach is only suitable for small quantum systems, for the number of measured observables grows exponentially with the dimension of the system [38].

In 1996, Bennett et al. proposed an entanglement measurement method named entanglement of formation (EOF) [41,42]. For a general quantum system consisting of two parts, here named A and B, an arbitrary pure state can be written as [43]:

$$
|\Phi\rangle=\sum_{i=1}^{n} c_{i}\left|\phi_{i}^{A}\right\rangle \otimes\left|\phi_{i}^{B}\right\rangle
$$

Here, $\left\{\left|\phi_{1}^{A}\right\rangle, \ldots,\left|\phi_{n}^{A}\right\rangle\right\}$ and $\left\{\left|\phi_{1}^{B}\right\rangle, \ldots,\left|\phi_{n}^{B}\right\rangle\right\}$ are sets of orthonormal states for A and B, respectively. The $c_{i}$ 's are a set of positive coefficients. The values of $c_{i}$ precisely show the features of the state $|\Phi\rangle$ that do not change when the parts of the system are subjected to separate unitary transformations. Therefore, the coefficients $c_{i}$ 's are of vice importance and any reasonable definition of the entanglement of $|\Phi\rangle$ should depend only on those values. In this way, the EOF for the state in Equation (1) is defined as:

$$
E(\Phi)=S\left(\operatorname{Tr}_{B}|\Phi\rangle\langle\Phi|\right)=S\left(\operatorname{Tr}_{A}|\Phi\rangle\langle\Phi|\right)=-\sum_{i}^{n} c_{i}^{2} \log _{2} c_{i}^{2},
$$

where $S$ means the von Neumann entropy. For describing the significance of the EOF, we take a particular entangled state, the singlet state $\left|\Psi^{-}\right\rangle=1 / \sqrt{2}(|01\rangle-|10\rangle)$, as the standard state and define its entanglement to be one "ebit". Suppose the two parties Alice and Bob want to create $n$ copies of $|\Phi\rangle$; they will need $n E(\Phi)$ singlet pairs in total [41].

On the other hand, the definition of EOF can be extended to a mixed state. Under this case, the EOF is designed to pick out the irreducible entanglement of the mixed state. Suppose Alice and Bob hope to create a mixed state $\rho$. They first write down a decomposition of $\rho$ into pure states as:

$$
\rho=\sum_{j=1}^{N} p_{j}\left|\Phi_{j}\right\rangle\left\langle\Phi_{j}\right|
$$


where $\left|\Phi_{j}\right\rangle$ is the distinct (but not necessarily orthogonal) normalized pure states of the bipartite system and $p_{j}$ is the non-negative real coefficient. The number of singlet pairs they needed can be written as:

$$
\text { number of singlet pairs }=\sum_{j=1}^{N} p_{j} E\left(\Phi_{j}\right)
$$

This number largely depends on the particular decomposition of $\rho$ that was chosen. The EOF of the state in Equation (3) is defined as the minimum number of the singlet pairs required to create $\rho$. In this way, for a mixed state in Equation (3), the EOF is:

$$
E_{f}(\rho)=i n f \sum_{j} p_{j} E\left(\Phi_{j}\right)
$$

corresponding to the decomposition that minimizes the expression in Equation (4).

It was proven that there is a general formula of $E_{f}[42,44,45]$, which is based on an exactly calculable quantity, that is concurrence (C). For a pure state $|\Phi\rangle$ in a two-qubit system, $C(|\Phi\rangle)$ is defined as $C(|\Phi\rangle)=|\langle\Phi \mid \widetilde{\Phi}\rangle|$. The $|\widetilde{\Phi}\rangle=\left(\sigma_{y} \otimes \sigma_{y}\right)\left|\Phi^{*}\right\rangle$, where $\sigma_{y}$ is the Pauli operator $\left(\begin{array}{cc}0 & -i \\ i & 0\end{array}\right)$, and $\left|\Phi^{*}\right\rangle$ is the complex conjugate of $|\Phi\rangle$. For a two-qubit system, the relationship between the concurrence and EOF can be written as:

$$
E(|\Phi\rangle)=\varepsilon(C(|\Phi\rangle))
$$

where:

$$
\varepsilon(C)=-\frac{1+\sqrt{1-C^{2}}}{2} \log _{2} \frac{1+\sqrt{1-C^{2}}}{2}-\frac{1-\sqrt{1-C^{2}}}{2} \log _{2} \frac{1-\sqrt{1-C^{2}}}{2} .
$$

Similar to the EOF definition of a mixed state $\rho$, we can also define the concurrence of a mixed state as the average concurrence of an ensemble of the decomposition of $\rho=\sum_{j} p_{j}\left|\Phi_{j}\right\rangle\left\langle\Phi_{j}\right|$, which is the minimum value over all decompositions of $\rho$. In this way, the concurrence can be written as [46]:

$$
C(\rho)=\inf \sum_{j} p_{j} C\left(\Phi_{j}\right)
$$

According to [43], there always exists a decomposition of $\rho$ that achieves the minimum in Equation (8) with a set of pure states. Therefore, the function $\varepsilon(C(\rho))$ defined in Equation (7) also provides us the EOF as:

$$
E_{f}(\rho)=\varepsilon(C(\rho)) .
$$

On the other hand, according to [45], there is an explicit formula for concurrence as:

$$
C(\rho)=\max \left\{0, \lambda_{1},-\lambda_{2},-\lambda_{3},-\lambda_{4}\right\} .
$$

In Equation (10), $\lambda_{i}(i=1,2,3,4)$ is the non-negative eigenvalue of the Hermitian matrix $R=\sqrt{\sqrt{\rho} \widetilde{\rho} \sqrt{\rho}}$ in decreasing order. Here, $\widetilde{\rho}=\left(\sigma_{y} \otimes \sigma_{y}\right) \rho^{*}\left(\sigma_{y} \otimes \sigma_{y}\right)$, and $\rho^{*}$ is the complex conjugate of $\rho$. It can be found that Equation (10) can reduce to the concurrence of the pure state formula $C(\Phi)=|\langle\Phi \mid \widetilde{\Phi}\rangle|$ when $\rho$ is a pure state as $|\Phi\rangle\langle\Phi|$. 
If we express the state $|\Phi\rangle$ in the standard basis as:

$$
|\Phi\rangle=c_{0}|00\rangle+c_{1}|01\rangle+c_{2}|10\rangle+c_{3}|11\rangle
$$

where $\left|c_{0}\right|^{2}+\left|c_{1}\right|^{2}+\left|c_{2}\right|^{2}+\left|c_{3}\right|^{2}=1$, the connection between concurrence and entanglement of a two-qubit system can be particularly clear. It can be found that $|\Phi\rangle$ is factorizable only under the case that $c_{0} c_{3}=c_{1} c_{2}$. Therefore, we can take the difference between $c_{0} c_{3}$ and $c_{1} c_{2}$ as a measurement of entanglement. In this way, we can obtain a more clear definition of the concurrence as $[43,45,47]$ :

$$
C(|\Phi\rangle)=2\left|c_{0} c_{3}-c_{1} c_{2}\right|
$$

Based on Equation (12), one can directly measure the concurrence of pure states by detecting only one single factorizable observable, which indicates that two copies of the composite state are available. In this way, some efficient concurrence measurement protocols based on a linear optical system or a nonlinear optical system have been proposed [48-57].

The review is organized as follows: Section 2 will explain some concurrence measurement protocols for the optical states. Section 3 will explain some concurrence measurement protocols for the atomic state. Section 4 will make a brief discussion and a conclusion.

\section{The Concurrence Measurement for the Optical States}

In 2006, Walborn et al. first experimentally realized the direct concurrence measurement of an arbitrary pure momentum photon stateas $\alpha|01\rangle_{a b}+\beta|10\rangle_{a b}$ [48,49]. Here, $a$ and $b$ represent two spatial modes, while " 1 " and " 0 " represent one and zero photons, respectively. The measurement process relies on the hyperentanglement. The experimental setup of the protocol is shown in Figure 1. In the protocol, two copies of a bipartite quantum state are created using a photon pair, which are generated in the spontaneous parametric down-conversion source. The polarization degree of freedom is stored in one copy of the quantum state, and the momentum degree of freedom is stored in the other copy. Owing to momentum conservation, the momentum states can be generated with neutral filters, phase plates and beam splitters, which combine different momentum modes [58-60]. Meanwhile, an arbitrary pure polarization state can be prepared by pumping two perpendicular nonlinear crystals with the additional help of the half and quarter wave plates placed in the path of both of the pump beams [61].

In the experimental process, all of the measurements focus on only a single photon, for example the first photon, which can significantly facilitate the experimental operation. According to the description above, a hyperentangled state of the first photon can be generated as:

$$
|\Phi\rangle=\left(\alpha|01\rangle_{a b}+\beta|10\rangle_{a b}\right) \otimes(\beta|H\rangle \pm \alpha|V\rangle)
$$

where $|H\rangle$ and $|V\rangle$ represent the horizontal and vertical polarization state, respectively, and $|a\rangle$ and $|b\rangle$ represent two different momentum modes. In this way, the first photon may be in four possible Bell states as:

$$
\begin{aligned}
\left|\psi^{ \pm}\right\rangle & =\alpha \beta(|H\rangle|b\rangle \pm|V\rangle|a\rangle), \\
\left|\phi^{ \pm}\right\rangle & =\alpha^{2}|H\rangle|a\rangle \pm \beta^{2}|V\rangle|b\rangle,
\end{aligned}
$$


It can be found that the success probability for obtaining $\left|\psi^{-}\right\rangle$or $\left|\psi^{+}\right\rangle$is $P_{A}=2|\alpha \beta|^{2}$. On the other hand, based on Equation (12), the theoretical value of concurrence of $\alpha|01\rangle_{a b}+\beta|10\rangle_{a b}$ is $C=2|\alpha \beta|$. Therefore, the concurrence can be determined by the success probability for obtaining the first photon in the antisymmetric state as $\left|\psi^{ \pm}\right\rangle$.

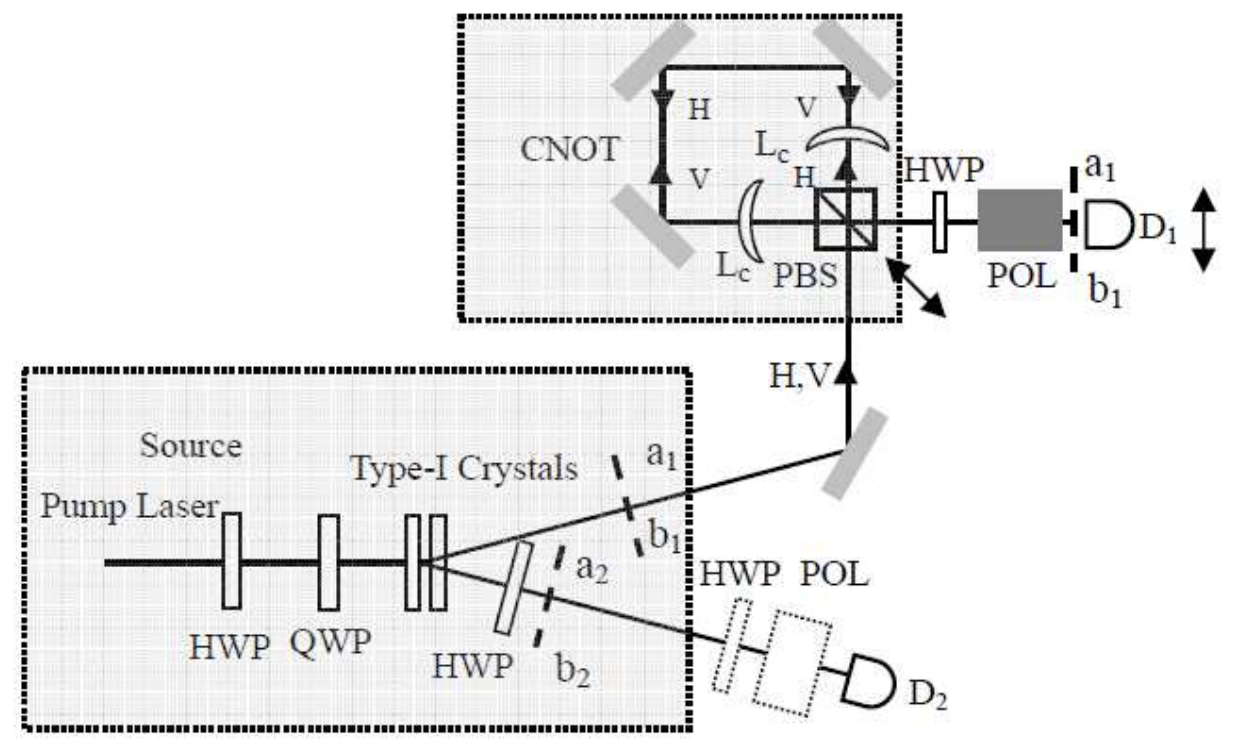

Figure 1. The experimental setup of the concurrence measurement protocol for the pure momentum entangled state as $\alpha|01\rangle_{a b}+\beta|10\rangle_{a b}$ in [48,49]. Two photon pairs, which bear entanglement in polarization and momentum degrees of freedom, respectively, can be created by the setup of Source. $D 1$ and $D 2$ represent the single photon detectors. HWP is the half-wave plate, and QWP is the quarter-wave plate. PBS is the polarizing beam splitter, and LC is the cylindrical lens. POL represents the polarization filter, while CNOT means the controlled-not gate. $\mathrm{H}$ and $\mathrm{V}$ indicate horizontal and vertical polarization, respectively.

The process to pick up $\left|\psi^{ \pm}\right\rangle$is as follows. The key setup is the interferometer, which is constructed by the polarizing beam splitter (PBS) and three focal length cylindrical lenses. According to Figure 1, the input photon first enters a polarizing beam splitter (PBS), which can transmit the photon in $|H\rangle$ and reflect the photon in $|V\rangle$. After the PBS, with the help of three 150-mm focal length cylindrical lenses, which are rotated by $45^{\circ}$ and $-45^{\circ}$, respectively, the photons in $|H\rangle$ and $|V\rangle$ will propagate in opposite directions within the interferometer and leave through the same exit port. As the photons in $|H\rangle$ and $|V\rangle$ encounter the lenses oriented at different angles, the resulting image of the photon in $|H\rangle$ is rotated by $90^{\circ}$, while that of the photon in $|V\rangle$ is rotated by $-90^{\circ}$, respectively. The interferometer performs the so-called controlled-not gate (CNOT) operation, for the momentum state of the output states will depend on the polarization [62]. If the photon is in $|V\rangle,|a\rangle$ will evolve to $|b\rangle$, and vice versa. If the photon is in $|H\rangle$, the momentum states will remain unchanged. In this way, after the CNOT operation, the four Bell states in Equation (14) can be transformed to:

$$
\begin{aligned}
& \left|\psi^{ \pm}\right\rangle \rightarrow \frac{1}{\sqrt{2}}(|H\rangle \pm|V\rangle)|b\rangle=| \pm\rangle|b\rangle, \\
& \left|\phi^{ \pm}\right\rangle \rightarrow \frac{1}{\sqrt{2}}(|H\rangle \pm|V\rangle)|a\rangle=| \pm\rangle|a\rangle .
\end{aligned}
$$


Therefore, the success probability for obtaining $\left|\psi^{ \pm}\right\rangle$equals that of obtaining $| \pm\rangle|b\rangle$. Then, the parties make the output photons from the PBS pass through another half-wave plate and polarization analyzer to discriminate the different polarization states. Meanwhile, they position two single photon detectors in the paths of Modes a and $b$ to discriminate the momentum state. In this way, the success possibility for obtaining $| \pm\rangle|b\rangle$ can be easily measured.

In 2013, the group of Cao proposed three theoretical protocols to measure the concurrence of a special two-photon polarization-entangled state as $|\psi\rangle=\beta|H H\rangle+\gamma|V V\rangle$, where $|\beta|^{2}+|\gamma|^{2}=1$ [50].

The first protocol is in linear optics, as shown in Figure 2. Two identical signal photon pairs are prepared in the special two-photon polarization-entangled pure states as:

$$
\begin{aligned}
\left|\psi_{1}\right\rangle_{a d_{1}} & =\beta|H H\rangle_{a d_{1}}+\gamma|V V\rangle_{a d_{1}} \\
\left|\psi_{2}\right\rangle_{b c_{1}} & =\beta|H H\rangle_{b c_{1}}+\gamma|V V\rangle_{b c_{1}}
\end{aligned}
$$

The parties make the photons in $c_{1}$ and $d_{1}$ modes pass through the 50:50 beam splitter (BS). Then, the whole four-photon system can evolve to:

$$
\begin{aligned}
\left|\psi_{1}\right\rangle_{a d_{1}} \otimes\left|\psi_{2}\right\rangle_{b c_{1}} \rightarrow & \frac{\beta^{2}}{\sqrt{2}}|H H\rangle_{a b} \otimes\left(|H H\rangle_{c}-|H H\rangle_{d}\right)+\frac{\gamma^{2}}{\sqrt{2}}|V V\rangle_{a b} \otimes\left(|V V\rangle_{c}-|V V\rangle_{d}\right) \\
& +\frac{\beta \gamma}{2}\left(|H V\rangle_{a b}+|V H\rangle_{a b}\right) \otimes\left(|H V\rangle_{c}-|H V\rangle_{d}\right) \\
& +\frac{\beta \gamma}{2}\left(|H V\rangle_{a b}-|V H\rangle_{a b}\right) \otimes\left(|H V\rangle_{c d}-|V H\rangle_{c d}\right) .
\end{aligned}
$$

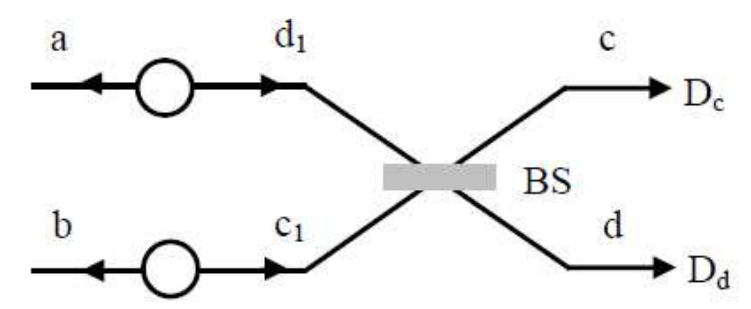

Figure 2. Schematic drawing of the first protocol of [50] with linear optics. Two pairs of two-photon purification-entangled states are required. The BS represents the 50:50 beam splitter. $D_{c}$ and $D_{d}$ are the single photon detectors.

The output photons in the spatial Modes $\mathrm{c}$ and $\mathrm{d}$ are detected by the single photon detectors $D_{c}$ and $D_{d}$, respectively. The parties select the items that make both of the two detectors fire. In this way, the four-photon state in Equation (17) will collapse to the balanced states as:

$$
\left|\psi_{3}\right\rangle_{a b}=|H V\rangle_{a b}-|V H\rangle_{a b}
$$

with the success probability of $P_{1}=|\beta \gamma|^{2}$.

According to Equations (11) and (12), $C\left(\left|\psi_{1}\right\rangle\right)=2|\beta \gamma|=2 \sqrt{P_{1}}$. Therefore, the concurrence of the two-photon polarization-entangled state can be directly measured by measuring the success probability for picking up the state of Equation (18). However, the detection efficiency of single-photon detectors is 
relatively low, and two of the four signal photons will be absorbed after the detection. For overcoming these two problems, in the second protocol, they adopt the cross-Kerr nonlinearity to realize the quantum nondemolition detection (QND) for the photons.

Before explaining the protocol in detail, we introduce the principle of the QND gate constructed by the cross-Kerr nonlinearity briefly [63-71]. The cross-Kerr nonlinearity can be described with its Hamiltonian [72]:

$$
H_{c k}=\hbar \chi \hat{n_{a}} \hat{n_{b}},
$$

where $\hbar \chi$ is the coupling strength of the nonlinearity, which depends on the cross-Kerr-material. $\hat{n_{a}}$ and $\hat{n_{b}}$ are the photon number operators for Modes $a$ and $b$. A coherent beam in the state $|\alpha\rangle$ and a single photon pulse with the form of $|\psi\rangle=\gamma|0\rangle+\delta|1\rangle$ interact with the cross-Kerr material. After the interaction, the system can evolve to:

$$
\left.U_{c k}|\psi| \alpha\right\rangle=(\gamma|0\rangle+\delta|1\rangle)|\alpha\rangle \rightarrow \gamma|0\rangle|\alpha\rangle+\delta|1\rangle\left|\alpha e^{i \theta}\right\rangle .
$$

Here, $|0\rangle$ and $|1\rangle$ mean no photon and one photon, respectively. $\theta=\chi t$, and $t$ means the interaction time for the signal photon with the nonlinear material. According to Equation (20), the phase shift of the coherent state is directly proportional to the photon number. By measuring the phase shift of the coherent state, one can judge the number of the signal photons without destroying the photon.

As shown in Figure 3, in the second protocol, the parties make the output photon in c and d modes pass through the cross-Kerr nonlinearities, which will make the coherent state $|\alpha\rangle$ pick up the phase shift of $\theta$ and $-\theta$, respectively. After the cross-Kerr nonlinearities, the four-photon state in Equation (17) will evolve to:

$$
\begin{aligned}
|\psi\rangle_{a b c d}= & \frac{\beta^{2}}{\sqrt{2}}|H H\rangle_{a b} \otimes\left(|H H\rangle_{c}\left|\alpha e^{i 2 \theta}\right\rangle-|H H\rangle_{d}\left|\alpha e^{-i 2 \theta}\right\rangle\right) \\
& +\frac{\gamma^{2}}{\sqrt{2}}|V V\rangle_{a b} \otimes\left(|V V\rangle_{c}\left|\alpha e^{i 2 \theta}\right\rangle-|V V\rangle_{d}\left|\alpha e^{-i 2 \theta}\right\rangle\right) \\
& +\frac{\beta \gamma}{2}\left(|H V\rangle_{a b}+|V H\rangle_{a b}\right) \otimes\left(|H V\rangle_{c}\left|\alpha e^{i 2 \theta}\right\rangle-|H V\rangle_{d}\left|\alpha e^{-i 2 \theta}\right\rangle\right) \\
& +\frac{\beta \gamma}{2}\left(|H V\rangle_{a b}-|V H\rangle_{a b}\right) \otimes\left(|H V\rangle_{c d}-|V H\rangle_{c d}\right)|\alpha\rangle
\end{aligned}
$$

Then, the parties choose the items corresponding to the zero phase shift in the coherent state $|\alpha\rangle$. Under this case, they can also pick up the state in Equation (18) with the probability of $P_{2}=|\beta \gamma|^{2}$.

In the protocol, the complex single photon detectors are not required. Moreover, after the detection, the photons in the $\mathrm{c}$ and $\mathrm{d}$ modes can be remained for other applications. Both of the two above protocols can also be used to measure the concurrence of general two-photon polarization pure state as $|\varphi\rangle=$ $c_{0}|H H\rangle+c_{1}|H V\rangle+c_{2}|V H\rangle+c_{3}|V V\rangle$. However, they cannot be used to measure the concurrence of the mixed state. The third protocol is an improvement of the second one, which can directly measure the concurrence of both pure and mixed states.

In the third protocol, suppose that two slightly different copies of the mixed photon states are prepared as $[73,74]$ :

$$
\begin{aligned}
\rho_{12}(F) & =F|\psi\rangle\langle\psi|+(1-F)| H V\rangle\langle V H|, \\
\rho_{34}\left(F^{\prime}\right) & =F^{\prime}|\psi\rangle\left\langle\psi\left|+\left(1-F^{\prime}\right)\right| H V\right\rangle\langle V H| .
\end{aligned}
$$


where $|\psi\rangle$ is the state in Equation (16). The concurrence of the mixed state is $C[\rho(F)]=2 F|\beta \gamma|$.

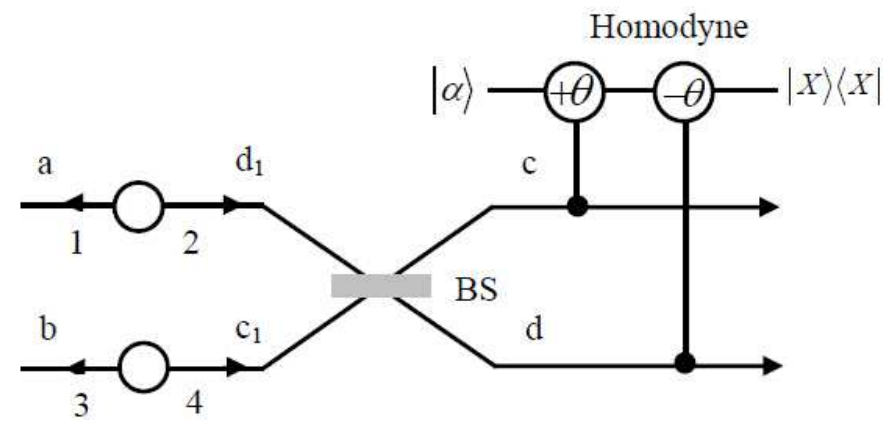

Figure 3. Schematic drawing of the second protocol in [50] with the cross-Kerr nonlinearities. The single photon in the $\mathrm{c}$ and $\mathrm{d}$ modes will make the coherent state $|\alpha\rangle$ pick up the phase shift of $\theta$ and $-\theta$, respectively.

Figure 4 shows the principle of the third protocol. Comparing with the second protocol, another cross-Kerr nonlinearity was adopted. The parties make the photons in the a1 and b1 modes pass through BS2, and the photons in $\mathrm{c} 1$ and $\mathrm{d} 1$ modes pass through BS1, simultaneously. Then, the output photons in the $\mathrm{a}$ and $\mathrm{b}$ and the $\mathrm{c}$ and $\mathrm{d}$ modes enter two QND gates, respectively. After the interaction, if both the two coherent states pick up no phase shift, they can select the balanced state at the output Modes c, $d$ of BS1 and a, b of BS2 as:

$$
\frac{F F^{\prime}|\beta \gamma|^{2}}{4}\left(|H V\rangle_{a b}-|V H\rangle_{a b}\right)\left(_ { a b } \left\langleH V | - { } _ { a b } \langle H V | ) \otimes ( | H V \rangle _ { c d } - | V H \rangle _ { c d } ) \left({ }_{c d}\langle H V|-{ }_{c d}\langle H V),\right.\right.\right.
$$

with the probability of $P_{3}=F F^{\prime}|\beta \gamma|^{2}$.

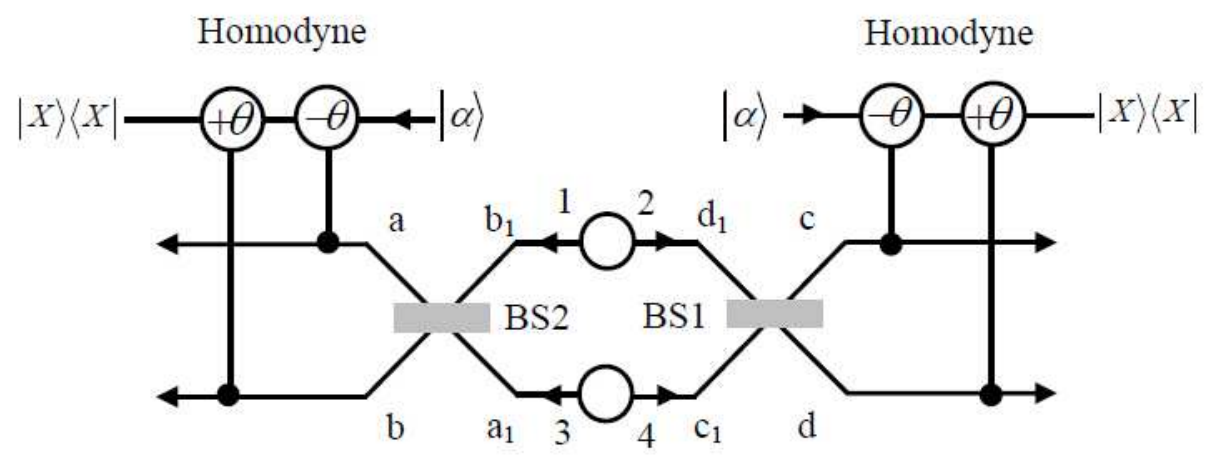

Figure 4. Schematic drawing of the third protocol in [50]. The parties also make photons in $\mathrm{a}$ and $\mathrm{b}$ modes pass through the cross-Kerr nonlinearities. The single photons in a and c modes will make the coherent state $|\alpha\rangle$ pick up the phase shift of $-\theta$, while the single photons in the $\mathrm{b}$ and $\mathrm{d}$ modes will make it pick up $\theta$.

Actually, Under this case, the obtained concurrence is $C_{m}=2 \sqrt{P_{3}}=2 \sqrt{F F^{\prime}}|\beta \gamma|=$ $\sqrt{F / F^{\prime}} C[\rho(F)]$. As the difference between $F$ and $F^{\prime}$ is slight, $F / F^{\prime}$ only slightly deviates from one. If the two copies $\rho_{12}(F)$ and $\rho_{34}\left(F^{\prime}\right)$ are identical, that is $F=F^{\prime}$, the concurrence actually obtained of the mixed state equals $C((F))=2 F|\beta \gamma|$. 
In the same year, the group of Cao put forward another protocol for directly measuring the concurrence of arbitrary two-photon polarization-entangled pure state based on the parity-check measurement (PCM) [51]. The structure of the PCM gate is shown in Figure 5. This PCM gate also resorts to the cross-Kerr nonlinear interaction and can distinguish the superposition and the mixtures of the states $|H V\rangle$ and $|V H\rangle$ from $|H H\rangle$ and $|V V\rangle$. Suppose the parties prepare two single photon states in the spatial Modes $a$ and $b$ as:

$$
|\psi\rangle_{a}=d_{1}|H\rangle_{a}+d_{2}|V\rangle_{a}, \quad|\psi\rangle_{b}=d_{3}|H\rangle_{b}+d_{4}|V\rangle_{b}
$$

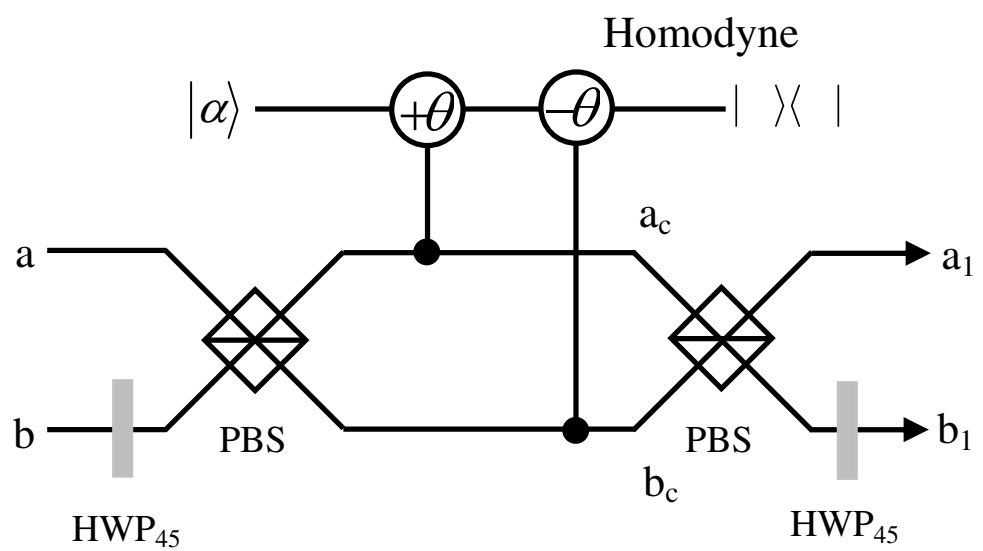

Figure 5. The structure of the PCM gate in [51]. The PBS can transmit the $|H\rangle$ polarized photon and reflect the $|V\rangle$ polarized photon. $H W P_{45}$ represents the half-wave plate oriented at $45^{\circ}$, which can make $|H\rangle \leftrightarrow|V\rangle$. The output photons from the PBS enter the cross-Kerr nonlinear media in the spacial modes of $a_{c}$ and $b_{c}$. The single photons in $a_{c}$ and $b_{c}$ modes can make the coherent state $|\alpha\rangle$ pick up the phase shift of $\theta$ and $-\theta$, respectively.

The parties make the two single photons pass through the PCM gate. The PBS can transmit the photon in $|H\rangle$ and reflect the photon in $|V\rangle . H W P_{45}$ is the half-wave plate oriented at $45^{\circ}$, which can make $|H\rangle \leftrightarrow|V\rangle$. The output photons from the left PBS enters the cross-Kerr nonlinear medias in the $a_{c}$ and $b_{c}$ modes, which will make the coherent state $|\alpha\rangle$ pick up the phase shift of $\theta$ and $-\theta$, respectively. The phase shifts $\pm \theta$ are undistinguishable in the homodyne measurement. After the PCM gate, the whole two-photon system combined with the coherent state $|\alpha\rangle$ will evolve to:

$$
\begin{aligned}
|\psi\rangle_{a} \otimes|\psi\rangle_{b} \otimes|\alpha\rangle \rightarrow & \left(d_{1} d_{4}|H V\rangle_{a_{1} b_{1}}+d_{2} d_{3}|V H\rangle_{a_{1} b_{1}}\right)|\alpha\rangle \\
& +\left(d_{1} d_{3}|H H\rangle_{a_{1} b_{1}}\left|\alpha e^{-2 i \theta}\right\rangle+d_{2} d_{4}|V V\rangle_{a_{1} b_{1}}\left|\alpha e^{2 i \theta}\right\rangle\right) .
\end{aligned}
$$

In this way, one can distinguish the odd parity states $|H V\rangle$ and $|V H\rangle$ from the even parity states $|H H\rangle$ and $|V V\rangle$ according to the different phase shifts of the coherent state.

The schematic principle of the protocol is shown in Figure 6. In the protocol, it requires two copies of arbitrary two-photon polarization-entangled states, say $\left|\phi_{12}\right\rangle$ and $\left|\phi_{34}\right\rangle$ with the form of:

$$
\left|\phi_{12(34)}\right\rangle=c_{0}|H H\rangle+c_{1}|H V\rangle+c_{2}|V H\rangle+c_{3}|V V\rangle \text {. }
$$


The parties firstly make Photons 2 and 4 pass through the PCM1, and Photons 1 and 3 pass through the PCM2, respectively. After the parity-check measurement, they pick up both the odd states of Photons 2, 4 and 1,3 . In this way, the whole four-photon system will collapse to:

$$
\left|\phi_{\text {odd } 1}\right\rangle_{1324}=\frac{1}{\sqrt{N}}\left[c_{0} c_{3}\left(|H V H V\rangle_{1324}+|V H V H\rangle_{1324}\right)+c_{1} c_{2}\left(|H V V H\rangle_{1324}+|V H H V\rangle_{1324}\right)\right]
$$

where the $N=2\left(\left|c_{0} c_{3}\right|^{2}+\left|c_{1} c_{2}\right|^{2}\right)$. The success probability to obtain the state of Equation (27) is $P_{1}=N$.

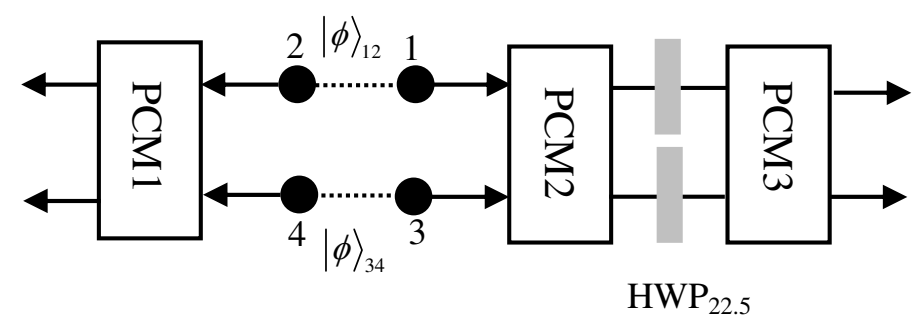

Figure 6. Schematic drawing of the protocol for directly measuring the concurrence of arbitrary two-photon polarization entangled states in [51]. The structure of Parity-Check Measurement 1 (PCM1), PCM2 and PCM3 is shown in Figure 5. $H W P_{22.5}$ is the half-wave plate oriented at $22.5^{\circ}$, which can make $|H\rangle \rightarrow(|H\rangle+|V\rangle) / \sqrt{2}$ and $|V\rangle \rightarrow(|H\rangle-|V\rangle) / \sqrt{2}$.

Then, they make Photons 1 and 3 pass through the $H W P_{22.5}$, which can make $|H\rangle \rightarrow(|H\rangle+|V\rangle) / \sqrt{2}$ and $|V\rangle \rightarrow(|H\rangle-|V\rangle) / \sqrt{2}$. After the $H W P_{22.5}$, the state of Equation (27) will evolve to:

$$
\begin{aligned}
\left|\phi_{H W P}\right\rangle= & \frac{\left(c_{1} c_{2}+c_{0} c_{3}\right)}{2 \sqrt{N}}\left(|H H\rangle_{13}-|V V\rangle_{13}\right) \otimes\left(|H V\rangle_{24}+|V H\rangle_{24}\right) \\
& +\frac{\left(c_{1} c_{2}-c_{0} c_{3}\right)}{2 \sqrt{N}}\left(|H V\rangle_{13}-|V H\rangle_{13}\right) \otimes\left(|H V\rangle_{24}-|V H\rangle_{24}\right) .
\end{aligned}
$$

Finally, the parties make Photons 1 and 3 pass through PCM3 and pick up the odd parity state of Photons 1 and 3 again. In this way, the state in Equation (28) will collapse to:

$$
\left|\phi_{\text {odd } 2}\right\rangle_{1324}=\frac{1}{2}\left(|H V\rangle_{13}-|V H\rangle_{13}\right) \otimes\left(|H V\rangle_{24}-|V H\rangle_{24}\right)
$$

with the success probability $P_{2}=\frac{\left|c_{0} c_{3}-c_{1} c_{2}\right|^{2}}{N}$.

It can be found that both the Photon Pairs 1, 3 and 2, 4 are the maximally-entangled states, which can be kept for further applications. The total success probability to obtain the state in Equation (29) is the product of the success probabilities in the above two steps, that is $P=P_{1} P_{2}=\left|c_{0} c_{3}-c_{1} c_{2}\right|^{2}$.

In this way, we can finally obtain the concurrence of the arbitrary two-photon polarization-entangled state as:

$$
C(|\phi\rangle)=2\left|c_{0} c_{3}-c_{1} c_{2}\right|=2 \sqrt{P} .
$$

Besides the cross-Kerr nonlinearity, some other nonlinear elements can also be used to realize the concurrence measurement of photon states. In 2013, Zhou proposed a concurrence measurement protocol for an arbitrary two-photon entanglement state with the help of the photonic Faraday rotation [52]. 
The basic principle of the photonic Faraday rotation is shown in Figure 7 [75-81]. The parties make a three-level atom trap in the one-side low-quality (Q) cavity, where the states $\left|g_{L}\right\rangle$ and $\left|g_{R}\right\rangle$ represent the two Zeeman sub-levels of its degenerate ground state, and $|e\rangle$ represents the excited state. A single photon pulse with the form of $\left|\varphi_{i n}\right\rangle=\frac{1}{\sqrt{2}}(|L\rangle+|R\rangle)$ enters the cavity and reacts with the three-level atom, where $|L\rangle$ and $|R\rangle$ represent the left-circular polarization and right-circular polarization of the input photon, respectively. When the atom absorbs or emits a $|L\rangle(|R\rangle)$ polarized photon, it will cause the transitions $\left|g_{L}\right\rangle \leftrightarrow|e\rangle\left(\left|g_{R}\right\rangle \leftrightarrow|e\rangle\right)$.

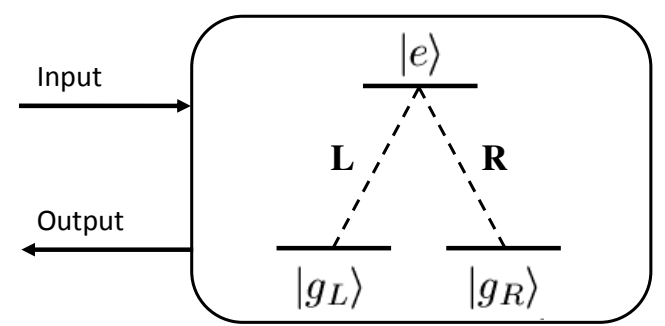

Figure 7. The interaction between the photon pulse and the three-level atom in the low-quality (Q) cavity. We make a three-level atom trap in a low-Q cavity. $\left|g_{L}\right\rangle$ and $\left|g_{R}\right\rangle$ represent the two Zeeman sub-levels of its degenerate ground state, and $|e\rangle$ represents its excited state. The state $\left|g_{L}\right\rangle$ and $\left|g_{R}\right\rangle$ couple with a left- (L) polarized and a right- (R) polarized photon, respectively.

When the photon pulse pass through the low-Q cavity, if the photon pulse takes action, the output photon state will convert to $\left|\varphi_{\text {out }}\right\rangle=r\left(\omega_{p}\right)|L(R)\rangle \simeq e^{i \phi}|L(R)\rangle$. Otherwise, the single photon would only sense the empty cavity, and the output photon state will convert to $\left|\varphi_{\text {out }}\right\rangle=r_{0}\left(\omega_{p}\right)|L(R)\rangle=e^{i \phi_{0}}|L(R)\rangle$.

In this way, for an input single-photon state $\left|\varphi_{i n}\right\rangle=\frac{1}{\sqrt{2}}(|L\rangle+|R\rangle)$, if the initial atomic state is $\left|g_{L}\right\rangle$, the output photon state can evolve to:

$$
\left|\varphi_{\text {out }}\right\rangle_{-}=\frac{1}{\sqrt{2}}\left(e^{i \phi}|L\rangle+e^{i \phi_{0}}|R\rangle\right)
$$

while if the initial atom state is $\left|g_{R}\right\rangle$, the output photon state will evolve to:

$$
\left|\varphi_{\text {out }}\right\rangle_{+}=\frac{1}{\sqrt{2}}\left(e^{i \phi_{0}}|L\rangle+e^{i \phi}|R\rangle\right) .
$$

Based on Equation (31) and Equation (32), the angle $\Theta_{F}^{-}=\phi_{0}-\phi$ or $\Theta_{F}^{+}=\phi-\phi_{0}$ is defined as the photonic Faraday rotation.

Therefore, under a special condition that $\phi=\pi$ and $\phi_{0}=\frac{\pi}{2}$, the relation between the input and output photon state can be rewritten as [79]:

$$
\begin{array}{rlrl}
|L\rangle\left|g_{L}\right\rangle & \rightarrow-|L\rangle\left|g_{L}\right\rangle, & |R\rangle\left|g_{L}\right\rangle & \rightarrow i|R\rangle\left|g_{L}\right\rangle, \\
|L\rangle\left|g_{R}\right\rangle & \rightarrow i|L\rangle\left|g_{R}\right\rangle, \quad|R\rangle\left|g_{R}\right\rangle \rightarrow-|R\rangle\left|g_{R}\right\rangle .
\end{array}
$$

In the measurement protocol of [52], two nonlocal parties, Alice and Bob, share two pairs of arbitrary two-photon entangled states with the same form of:

$$
\begin{aligned}
\left|\phi_{1}\right\rangle_{a 1 b 1} & =\alpha|R R\rangle_{a 1 b 1}+\beta|R L\rangle_{a 1 b 1}+\gamma|L R\rangle_{a 1 b 1}+\delta|L L\rangle_{a 1 b 1} \\
\left|\phi_{2}\right\rangle_{a 2 b 2} & =\alpha|R R\rangle_{a 2 b 2}+\beta|R L\rangle_{a 2 b 2}+\gamma|L R\rangle_{a 2 b 2}+\delta|L L\rangle_{a 2 b 2}
\end{aligned}
$$


where $|\alpha|^{2}+|\beta|^{2}+|\gamma|^{2}+|\delta|^{2}=1$.

The protocol includes two steps. As shown in Figure 8, the two parties make three three-level atoms, here named "1", "2" and "3", with the same form of $|\varphi\rangle=\frac{1}{\sqrt{2}}\left(\left|g_{L}\right\rangle+\left|g_{R}\right\rangle\right)$ trap in three low-Q cavities, respectively. In the first step, they both make the photons in their hands pass through low-Q cavities and interact with Atoms 1 and 2, simultaneously. Based on Equation (33), the relation between the input and output photon-atom state can be written as:

$$
\begin{aligned}
& |R\rangle|R\rangle\left(\left|g_{L}\right\rangle+\left|g_{R}\right\rangle\right) \rightarrow|R\rangle|R\rangle\left(-\left|g_{L}\right\rangle+\left|g_{R}\right\rangle\right), \\
& |L\rangle|L\rangle\left(\left|g_{L}\right\rangle+\left|g_{R}\right\rangle\right) \rightarrow|L\rangle|L\rangle\left(\left|g_{L}\right\rangle-\left|g_{R}\right\rangle\right), \\
& |R\rangle|L\rangle\left(\left|g_{L}\right\rangle+\left|g_{R}\right\rangle\right) \rightarrow-i|R\rangle|L\rangle\left(\left|g_{L}\right\rangle+\left|g_{R}\right\rangle\right), \\
& |L\rangle|R\rangle\left(\left|g_{L}\right\rangle+\left|g_{R}\right\rangle\right) \rightarrow-i|L\rangle|R\rangle\left(\left|g_{L}\right\rangle+\left|g_{R}\right\rangle\right) .
\end{aligned}
$$

Then, each of them picks up the photon state in odd parity, which does not change the atomic state. Under this case, the state of the whole four photons will evolve to:

$$
\left|\Phi_{1}\right\rangle_{a 1 a 2 b 1 b 2}=\alpha \delta\left(|R L R L\rangle_{a 1 a 2 b 1 b 2}+|L R L R\rangle_{a 1 a 2 b 1 b 2}\right)+\beta \gamma\left(|R L L R\rangle_{a 1 a 2 b 1 b 2}+|L R R L\rangle_{a 1 a 2 b 1 b 2}\right),
$$

with the success probability of $P_{1}=2|\alpha \delta|^{2}+2|\beta \gamma|^{2}$.

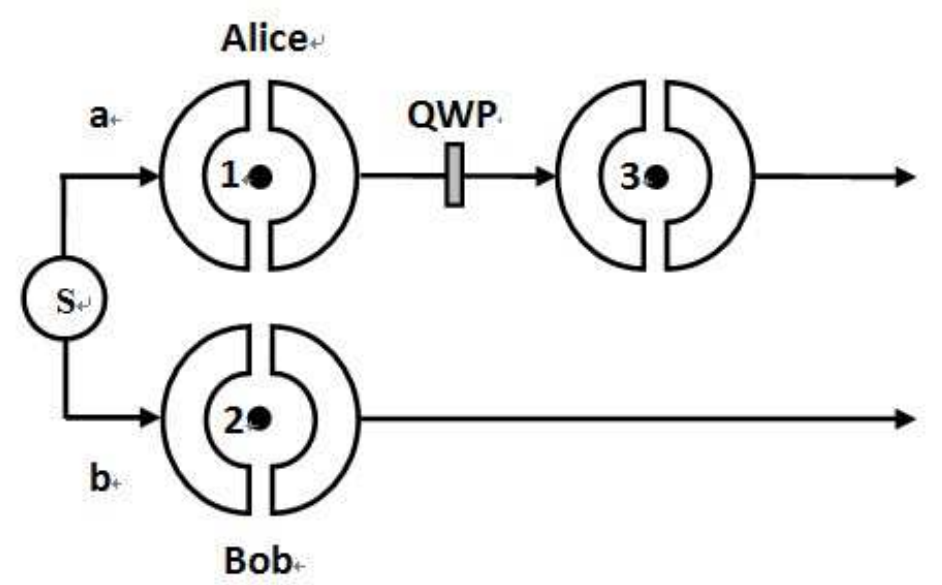

Figure 8. A schematic drawing of the concurrence measurement protocol for the arbitrary two-photon entanglement state in [52]. Two pairs of photonic states with the form of $|\phi\rangle=$ $\alpha|R R\rangle_{a b}+\beta|R L\rangle_{a b}+\gamma|L R\rangle_{a b}+\delta|L L\rangle_{a b}$ are generated by the single photon source $S$. The parties Alice and Bob make three three-level atoms with the same form of $\frac{1}{\sqrt{2}}\left(\left|g_{L}\right\rangle+\left|g_{R}\right\rangle\right)$ trap in three low-Q cavities, respectively. They make the photons in Modes a and b pass through the cavity and interact with the three-level atoms. The QWP represents the quarter wave plate.

Then, in the second step, Alice makes the photons in $a 1$ and $a 2$ modes pass through the quarter-wave plate (QWP), which can make $|R\rangle \rightarrow \frac{1}{\sqrt{2}}(|R\rangle+|L\rangle)$ and $|L\rangle \rightarrow \frac{1}{\sqrt{2}}(|R\rangle-|L\rangle)$. After that, the state of Equation (36) will evolve to:

$$
\begin{aligned}
\left|\Phi_{2}\right\rangle_{a 1 a 2 b 1 b 2}= & (\alpha \delta+\beta \gamma)(|R R\rangle-|L L\rangle)_{a 1 a 2}(|R L\rangle+|L R\rangle)_{b 1 b 2} \\
& +(\alpha \delta-\beta \gamma)(|L R\rangle-|R L\rangle)_{a 1 a 2}(|R L\rangle-|L R\rangle)_{b 1 b 2} .
\end{aligned}
$$


After the quarter-wave plate (QWP), Alice makes the two photons in her hand enter another cavity and interact with Atom " 3 ", and she also picks up the photon state in odd parity. In this way, the state of Equation (37) will finally evolve to:

$$
\left|\Phi_{3}\right\rangle_{a 1 a 2 b 1 b 2}=(|L R\rangle-|R L\rangle)_{a 1 a 2}(|R L\rangle-|L R\rangle)_{b 1 b 2}
$$

with the success probability of $P_{2}=\frac{|\alpha \delta-\beta \gamma|^{2}}{2\left(|\alpha \delta|^{2}+|\beta \gamma|^{2}\right)}$.

Based on the two steps described above, the total success probability for obtaining the state in Equation (38) can be calculated as:

$$
P_{\text {total }}=P_{1} P_{2}=|\alpha \delta-\beta \gamma|^{2}=\frac{C^{2}(|\phi\rangle)}{4} .
$$

Therefore, the concurrence of $\left|\phi_{1}\right\rangle_{a 1 b 1}$ can be easily obtained by measuring the success probability for obtaining the state of Equation (38).

In 2014, Sheng et al. proposed an effect concurrence measurement protocol for the hyperentangled state [57]. Considering an enlarged Hilbert space, the hyperentangled state can be regarded as the product of $N$ Bell states with the form of $[82,83]$ :

$$
|\Upsilon\rangle=\left|\Theta_{1}\right\rangle \otimes\left|\Theta_{2}\right\rangle \cdots\left|\Theta_{N}\right\rangle
$$

Here, $N$ denotes the degrees of freedom. Suppose that the concurrence of the Bell state $\left|\Theta_{i}\right\rangle$ is $C_{i}(i=1,2, \cdots N)$; the total concurrence of the hyperentangled state can be written as:

$$
C_{\text {hyper }}=\sum_{i=1}^{N} C_{N} .
$$

The protocol can be used to measure the concurrence of an arbitrary hyperentangled state with four different coefficients. In the protocol, the parties require two pairs of arbitrary polarization-momentum hyperentangled states with the form of:

$$
\begin{aligned}
|\phi\rangle= & |\phi\rangle_{p} \otimes|\phi\rangle_{m}=\left(\alpha_{1}\left|H_{a}\right\rangle\left|H_{b}\right\rangle+\beta_{1}\left|V_{a}\right\rangle\left|V_{b}\right\rangle+\gamma_{1}\left|H_{a}\right\rangle\left|V_{b}\right\rangle+\delta_{1}\left|V_{a}\right\rangle\left|H_{b}\right\rangle\right) \\
& \otimes\left(\alpha_{2}|a 1\rangle|b 1\rangle+\beta_{2}|a 2\rangle|b 2\rangle+\gamma_{2}|a 1\rangle|b 2\rangle+\delta_{2}|a 2\rangle|b 1\rangle\right),
\end{aligned}
$$

where $\left|\alpha_{1}\right|^{2}+\left|\beta_{1}\right|^{2}+\left|\gamma_{1}\right|^{2}+\left|\delta_{1}\right|^{2}=1$ and $\left|\alpha_{2}\right|^{2}+\left|\beta_{2}\right|^{2}+\left|\gamma_{2}\right|^{2}+\left|\delta_{2}\right|^{2}=1$.

The protocol includes two steps. The first step is used to measure the momentum entanglement. As shown in Figure 9, the parties make the photons in the $a_{1}, b_{1}, a_{3}$ and $b_{3}$ modes pass through two cross-Kerr materials with the coherent states of $|\tau\rangle_{A}$ and $|\tau\rangle_{B}$, respectively. If both of the coherent states pick up the phase shift of $\pm \theta$, the momentum entanglement state will become:

$$
\begin{aligned}
|\phi\rangle_{1}= & \frac{\alpha_{2} \beta_{2}}{\sqrt{2\left(\left|\alpha_{2} \beta_{2}\right|^{2}+\left|\gamma_{2} \delta_{2}\right|^{2}\right)}}(|a 1\rangle|a 4\rangle|b 1\rangle|b 4\rangle+|a 2\rangle|a 3\rangle|b 2\rangle|b 3\rangle) \\
& +\frac{\gamma_{2} \delta_{2}}{\sqrt{2\left(\left|\alpha_{2} \beta_{2}\right|^{2}+\left|\gamma_{2} \delta_{2}\right|^{2}\right)}}(|a 1\rangle|a 4\rangle|b 2\rangle|b 3\rangle+|a 2\rangle|a 3\rangle|b 1\rangle|b 4\rangle),
\end{aligned}
$$

with the success probability being $P_{1 m}=2\left(\left|\alpha_{2} \beta_{2}\right|^{2}+\left|\gamma_{2} \delta_{2}\right|^{2}\right)$. 


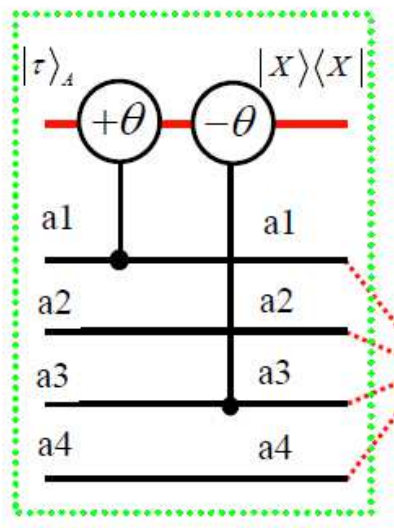

Alice

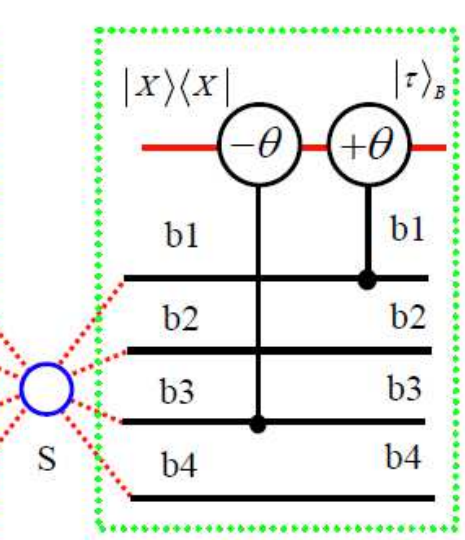

$\mathrm{Bob}$

Figure 9. The schematic of the concurrence measurement for the momentum entanglement. Two pairs of hyperentangled states are emitted by the source (S). This setup can make the parity check for the momentum entanglement.

Then, as shown in Figure 10, the parties make the photons in the spatial Modes $b 1, b 2, b 3$ and $b 4$ pass through two BSs, which can make the state $|\phi\rangle_{1}$ become:

$$
\begin{aligned}
|\phi\rangle_{1}^{\prime}= & \frac{\alpha_{2} \beta_{2}+\gamma_{2} \delta_{2}}{2 \sqrt{2\left(\left|\alpha_{2} \beta_{2}\right|^{2}+\left|\gamma_{2} \delta_{2}\right|^{2}\right)}}(|a 1\rangle|a 4\rangle+|a 2\rangle|a 3\rangle) \otimes\left(\left|c_{1}\right\rangle\left|c_{3}\right\rangle-\left|c_{2}\right\rangle\left|c_{4}\right\rangle\right) \\
& +\frac{\alpha_{2} \beta_{2}-\gamma_{2} \delta_{2}}{2 \sqrt{2\left(\left|\alpha_{2} \beta_{2}\right|^{2}+\left|\gamma_{2} \delta_{2}\right|^{2}\right)}}(|a 1\rangle|a 4\rangle-|a 2\rangle|a 3\rangle) \otimes(|c 1\rangle|c 4\rangle-|c 2\rangle|c 3\rangle) .
\end{aligned}
$$

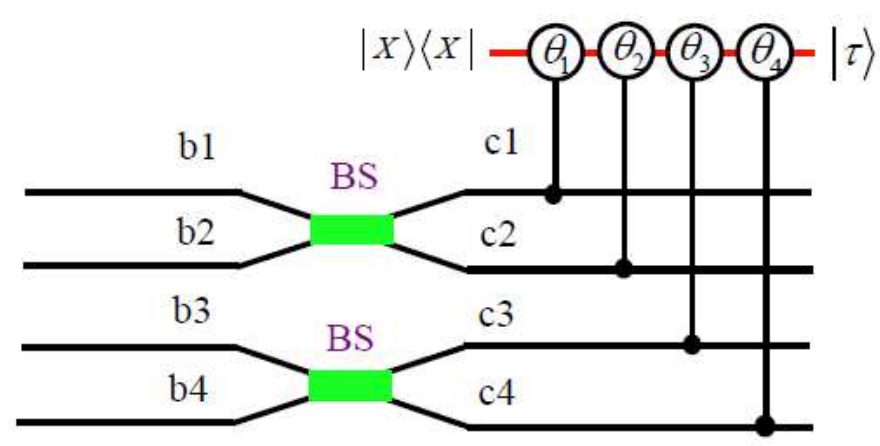

Figure 10. Schematic principle of the momentum entanglement measurement. In the last step, we should determine the spatial modes of the state to perform further measurement.

Next, the parties make the output photons in c1, c2, c3 and c4 modes pass through the cross-Kerr nonlinearities. Under the case that the coherent state picks up the phase shift of $\theta_{1}+\theta_{4},|\phi\rangle_{1}^{\prime}$ will become:

$$
|\phi\rangle_{1}^{\prime \prime}=\frac{1}{\sqrt{2}}(|a 1\rangle|a 4\rangle-|a 2\rangle|a 3\rangle) \otimes|c 1\rangle|c 4\rangle
$$

with the probability of $P_{2 m}=\frac{\left|\alpha_{2} \beta_{2}-\gamma_{2} \delta_{2}\right|^{2}}{4\left(\left|\alpha_{2} \beta_{2}\right|^{2}+\left|\gamma_{2} \delta_{2}\right|^{2}\right)}$. 
Based on the process above, the total probability for obtaining $|\phi\rangle_{1}^{\prime \prime}$ is:

$$
P_{m}=P_{1 m} P_{2 m}=2\left(\left|\alpha_{2} \beta_{2}\right|^{2}+\left|\gamma_{2} \delta_{2}\right|^{2}\right) \frac{\left|\alpha_{2} \beta_{2}-\gamma_{2} \delta_{2}\right|^{2}}{4\left|\alpha_{2} \beta_{2}\right|^{2}+\left|\gamma_{2} \delta_{2}\right|^{2}},=\frac{1}{2}\left|\alpha_{2} \beta_{2}-\gamma_{2} \delta_{2}\right|^{2} .
$$

and we can obtain the concurrence of the momentum entanglement as:

$$
C\left(|\phi\rangle_{m}\right)=2\left|\alpha_{2} \beta_{2}-\gamma_{2} \delta_{2}\right|=2 \sqrt{2 P_{m}}
$$

In the second step, by only considering the polarization entanglement, $|\phi\rangle_{1}^{\prime \prime}$ can be rewritten as:

$$
\begin{aligned}
|\psi\rangle_{1}= & \left(\alpha_{1}\left|H_{a}\right\rangle\left|H_{c}\right\rangle+\beta_{1}\left|V_{a}\right\rangle\left|V_{c}\right\rangle+\gamma_{1}\left|H_{a}\right\rangle\left|V_{c}\right\rangle+\delta_{1}\left|V_{a}\right\rangle\left|H_{c}\right\rangle\right) \\
& \otimes\left(\alpha_{1}\left|H_{a}\right\rangle\left|H_{c}\right\rangle+\beta_{1}\left|V_{a}\right\rangle\left|V_{c}\right\rangle+\gamma_{1}\left|H_{a}\right\rangle\left|V_{c}\right\rangle+\delta_{1}\left|V_{a}\right\rangle\left|H_{c}\right\rangle\right) \\
& \otimes \frac{1}{\sqrt{2}}(|a 1\rangle|a 4\rangle-|a 2\rangle|a 3\rangle) \otimes|c 1\rangle|c 4\rangle .
\end{aligned}
$$

The parties let the photons in $a 1, a 2, a 3$ and $a 4$ pass through the setup shown in Figure 10 by removing the two BSs. If the phase shift of the coherent state is $\theta_{1}+\theta_{4},|\psi\rangle_{1}$ will collapse to:

$$
\begin{aligned}
|\psi\rangle_{1}^{\prime}= & \left(\alpha_{1}\left|H_{a 1}\right\rangle\left|H_{c 1}\right\rangle+\beta_{1}\left|V_{a 1}\right\rangle\left|V_{c 1}\right\rangle+\gamma_{1}\left|H_{a 1}\right\rangle\left|V_{c 1}\right\rangle+\delta_{1}\left|V_{a 1}\right\rangle\left|H_{c 1}\right\rangle\right) \\
& \otimes\left(\alpha_{1}\left|H_{a 4}\right\rangle\left|H_{c 4}\right\rangle+\beta_{1}\left|V_{a 4}\right\rangle\left|V_{c 4}\right\rangle+\gamma_{1}\left|H_{a 4}\right\rangle\left|V_{c 4}\right\rangle+\delta_{1}\left|V_{a 4}\right\rangle\left|H_{c 4}\right\rangle\right) .
\end{aligned}
$$

After the measurement, the spatial mode of each photon is deterministic. Next, the parties can start the concurrence measurement of the polarization entanglement. Suppose each of the two parties owns a QND gate shown in Figure 11. They first let the four photons in their hands pass through the two QNDs, respectively, and pick up the case that both the two coherent states pick up the phase shift of $\pm 2 \theta$. Therefore, $|\psi\rangle_{1}^{\prime}$ will become:

$$
\begin{aligned}
|\psi\rangle_{2}^{\prime}= & \frac{\alpha_{1} \beta_{1}}{\sqrt{2\left(\left|\alpha_{1} \beta_{1}\right|^{2}+\left|\gamma_{1} \delta_{1}\right|^{2}\right)}}\left(\left|H_{a 1}\right\rangle\left|V_{a 4}\right\rangle\left|H_{c 1}\right\rangle\left|V_{c 4}\right\rangle+\left|V_{a 1}\right\rangle\left|H_{a 4}\right\rangle\left|V_{c 1}\right\rangle\left|H_{c 4}\right\rangle\right) \\
& +\frac{\gamma_{1} \delta_{1}}{\sqrt{2\left(\left|\alpha_{1} \beta_{1}\right|^{2}+\left|\gamma_{1} \delta_{1}\right|^{2}\right)}}\left(\left|H_{a 1}\right\rangle\left|V_{a 4}\right\rangle\left|V_{c 1}\right\rangle\left|H_{c 4}\right\rangle+\left|V_{a 1}\right\rangle\left|H_{a 4}\right\rangle\left|H_{c 1}\right\rangle\left|V_{c 4}\right\rangle\right)
\end{aligned}
$$

with the probability $P_{1 p}=2\left(\left|\alpha_{1} \beta_{1}\right|^{2}+\left|\gamma_{1} \delta_{1}\right|^{2}\right)$.

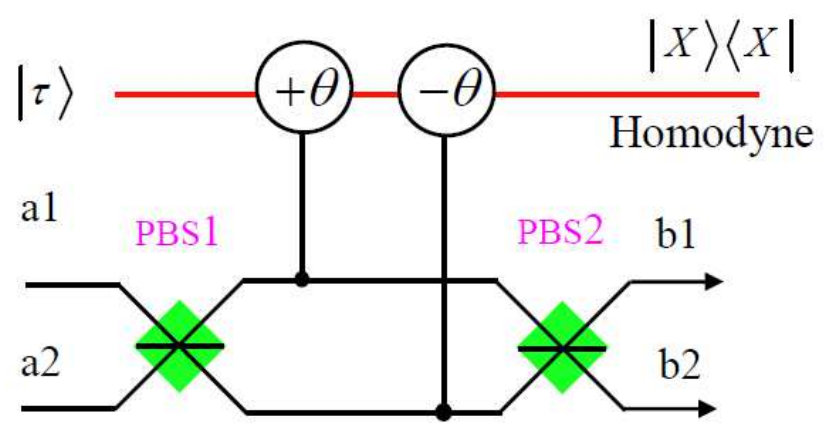

Figure 11. Schematic drawing of the parity check measurement for the polarization entanglement. 
Then, they perform the Hadamard operation on the photons in the $a 1$ and $a 4$ modes, which can make $|H\rangle \rightarrow \frac{1}{\sqrt{2}}(|H\rangle+|V\rangle),|V\rangle \rightarrow \frac{1}{\sqrt{2}}(|H\rangle-|V\rangle)$. After performing the Hadamard operation, the state $|\psi\rangle_{2}^{\prime}$ will become:

$$
\begin{aligned}
|\psi\rangle_{3}^{\prime}= & \frac{\alpha_{1} \beta_{1}+\gamma_{1} \delta_{1}}{2 \sqrt{2\left(\left|\alpha_{1} \beta_{1}\right|^{2}+\left|\gamma_{1} \delta_{1}\right|^{2}\right)}}\left(\left|H_{a 1}\right\rangle\left|H_{a 4}\right\rangle-\left|V_{a 1}\right\rangle\left|V_{a 4}\right\rangle\right) \otimes\left(\left|H_{c 1}\right\rangle\left|V_{c 4}\right\rangle+\left|V_{c 1}\right\rangle\left|H_{c 4}\right\rangle\right) \\
& +\frac{\alpha_{1} \beta_{1}-\gamma_{1} \delta_{1}}{2 \sqrt{2\left(\left|\alpha_{1} \beta_{1}\right|^{2}+\left|\gamma_{1} \delta_{1}\right|^{2}\right)}}\left(\left|H_{a 1}\right\rangle\left|V_{a 4}\right\rangle-\left|V_{a 1}\right\rangle\left|H_{a 4}\right\rangle\right) \otimes\left(\left|H_{c 1}\right\rangle\left|V_{c 4}\right\rangle-\left|V_{c 1}\right\rangle\left|H_{c 4}\right\rangle\right)
\end{aligned}
$$

Finally, they make the photons in the $a 1$ and $a 4$ modes pass through the QND gate shown in Figure 10 again and pick up the odd parity state for a second time, which can transform the state $|\psi\rangle_{3}^{\prime}$ to:

$$
|\psi\rangle_{4}^{\prime}=\frac{1}{2}\left(\left|H_{a 1}\right\rangle\left|V_{a 4}\right\rangle-\left|V_{a 1}\right\rangle\left|H_{a 4}\right\rangle\right) \otimes\left(\left|H_{c 1}\right\rangle\left|V_{c 4}\right\rangle-\left|V_{c 1}\right\rangle\left|H_{c 4}\right\rangle\right),
$$

with the probability $P_{2 p}=\frac{\left|\alpha_{2} \beta_{2}-\gamma_{2} \delta_{2}\right|^{2}}{2\left(\left|\alpha_{2} \beta_{2}\right|^{2}+\left|\gamma_{2} \delta_{2}\right|^{2}\right)}$.

Therefore, the total probability to obtain the $|\psi\rangle_{4}^{\prime}$ will be:

$$
P_{p}=P_{1 p} P_{2 p}=\left|\alpha_{1} \beta_{1}-\gamma_{1} \delta_{1}\right|^{2}
$$

We can obtain the concurrence of the polarization entanglement as:

$$
C\left(|\phi\rangle_{p}\right)=\left|\alpha_{2} \beta_{2}-\gamma_{2} \delta_{2}\right|=2 \sqrt{P_{p}}
$$

In this way, the total concurrence of the polarization-momentum hyperentanglement state can be written as:

$$
C(|\phi\rangle)=C\left(|\phi\rangle_{m}\right)+C\left(|\phi\rangle_{p}\right)=2 \sqrt{2 P_{m}}+2 \sqrt{P_{p}}
$$

\section{The Concurrence Measures for the Atomic State}

Besides the optical system, the concurrence measurement protocols for an atomic state have also been investigated. In 2007, with the help of the CNOT operation, Romero et al. proposed an efficient protocol for directly measuring an arbitrary two-qubit pure atomic state [53].

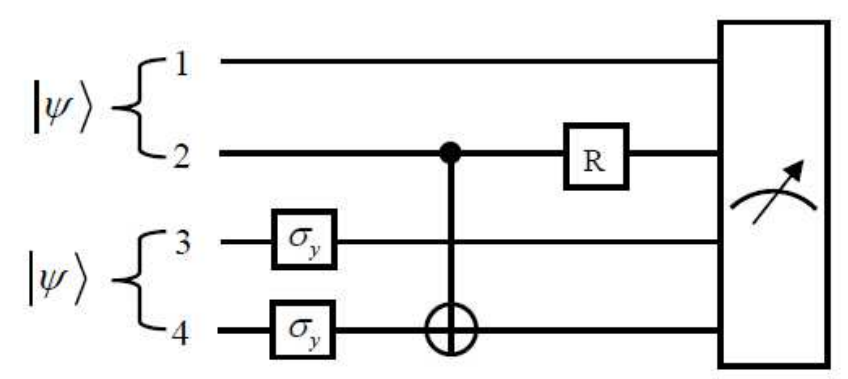

Figure 12. The direct measurement of the concurrence of a two-qubit pure atomic state in [53]. Two copies of atom states are required. The protocol involves a controlled-not (CNOT) gate. The $\sigma_{y}$ unitaries and other simple $\mathrm{R}$ qubit rotations, followed by the joint measurement of the four qubits. 
The schematic principle of the protocol is shown in Figure 12. It requires two copies of the two-qubit pure atom states with the same form of:

$$
|\psi\rangle=c_{0}|g g\rangle+c_{1}|g e\rangle+c_{2}|e g\rangle+c_{3}|e e\rangle
$$

where $|g\rangle$ and $|e\rangle$ represent the ground state and excited state of the two-level atom, respectively.

The parities need to take local operations on the second copy of the atom state as $|\Phi\rangle=|\psi\rangle \otimes\left(\sigma_{y} \otimes\right.$ $\left.\sigma_{y}|\psi\rangle\right)$. After the operation, the state of the whole four-atom system can be written as:

$$
\begin{aligned}
|\Phi\rangle= & \left.\left.\left.-c_{0} c_{3}|g g g g\rangle+c_{0} c_{2} \mid \text { ggge }\right\rangle+c_{0} c_{1} \mid \text { ggeg }\right\rangle-c_{1} c_{3} \mid \text { gegg }\right\rangle \\
& \left.\left.\left.\left.-c_{2} c_{3} \mid \text { eggg }\right\rangle-c_{0}^{2} \mid \text { ggee }\right\rangle-c_{3}^{2} \mid \text { eegg }\right\rangle+c_{1} c_{2} \mid \text { gege }\right\rangle \\
& \left.\left.\left.\left.+c_{1}^{2} \mid \text { geeg }\right\rangle+c_{2}^{2} \mid \text { egge }\right\rangle+c_{1} c_{2} \mid \text { egeg }\right\rangle-c_{0} c_{1} \mid \text { geee }\right\rangle \\
& \left.\left.\left.\left.-c_{0} c_{2} \mid \text { egee }\right\rangle+c_{2} c_{3} \mid \text { eege }\right\rangle+c_{1} c_{3} \mid \text { eeeg }\right\rangle-c_{0} c_{3} \mid \text { eeee }\right\rangle .
\end{aligned}
$$

Next, the parties take the CNOT operation between the second and the forth qubit. The second qubit acts as the control qubit, while the forth qubit acts as the target. If the control qubit is $|g\rangle$, the target will not be affected; conversely, if the control qubit is $|e\rangle$, the target will be flipped. Then, the parties apply the rotation $(\mathrm{R})$ operation on the second qubit, which makes:

$$
|g\rangle_{2} \rightarrow \frac{1}{\sqrt{2}}\left(|g\rangle_{2}-|e\rangle_{2}\right), \quad|e\rangle_{2} \rightarrow \frac{1}{\sqrt{2}}\left(|g\rangle_{2}+|e\rangle_{2}\right) .
$$

After the CNOT and R operations, the state in Equation (57) will evolve to:

$$
\begin{aligned}
\left|\Phi_{1}\right\rangle= & \left.\left.\frac{1}{\sqrt{2}}\left\{\left(c_{1} c_{2}-c_{0} c_{3}\right) \mid \text { gggg }\right\rangle+\left(c_{1} c_{2}+c_{0} c_{3}\right) \mid \text { gegg }\right\rangle+\left(c_{0} c_{2}-c_{1} c_{3}\right) \mid \text { ggge }\right\rangle \\
& \left.\left.\left.\left.-\left(c_{0} c_{2}+c_{1} c_{3}\right) \mid \text { gege }\right\rangle+2 c_{2} c_{3} \mid \text { eegg }\right\rangle-2 c_{0} c_{1} \mid \text { geeg }\right\rangle+\left(c_{1}^{2}-c_{0}^{2}\right) \mid \text { ggee }\right\rangle \\
& \left.\left.\left.\left.+\left(c_{1}^{2}+c_{0}^{2}\right) \mid \text { geee }\right\rangle+\left(c_{2}^{2}-c_{3}^{2}\right) \mid \text { egge }\right\rangle-\left(c_{2}^{2}+c_{3}^{2}\right) \mid \text { eege }\right\rangle+\left(c_{1} c_{2}-c_{0} c_{3}\right) \mid \text { egeg }\right\rangle \\
& \left.\left.\left.\left.-\left(c_{1} c_{2}+c_{0} c_{3}\right) \mid \text { eeeg }\right\rangle+\left(c_{0} c_{2}+c_{1} c_{3}\right) \mid \text { eeee }\right\rangle-\left(c_{0} c_{2}-c_{1} c_{3}\right) \mid \text { egee }\right\rangle\right\} .
\end{aligned}
$$

It can be found that the success probability for picking up $|g g g g\rangle$ or $\mid$ egeg $\rangle$ is $P_{g g g g}=P_{\text {egeg }}=$ $\left|c_{1} c_{2}-c_{0} c_{3}\right|^{2} / 2$. In this way, the concurrence information is present in the success probability for obtaining $|g g g g\rangle$ and $|e g e g\rangle$, which can be written as:

$$
C(|\psi\rangle)=2 \sqrt{2 P_{\text {gggg }}}=2 \sqrt{2 P_{\text {egeg }}} .
$$

Therefore, we can directly measure the concurrence of the two-qubit pure atomic state according to the success probability for obtaining the state $|g g g g\rangle$ or $\mid$ ege $\rangle\rangle$.

The experimental realization of the protocol is shown in Figure 13. The protocol can be applied to a two-cavity setup in microwave 3D cavity quantum electrodynamics (QED) and straightforwardly in trapped ion systems. The first cavity $\mathrm{C}$ is used to create two copies of identical entangled two-atom states with an entangling technique [84,85], and the second cavity D is used to realize the CNOT control [86]. The detailed experimental operations are shown in [53]. 


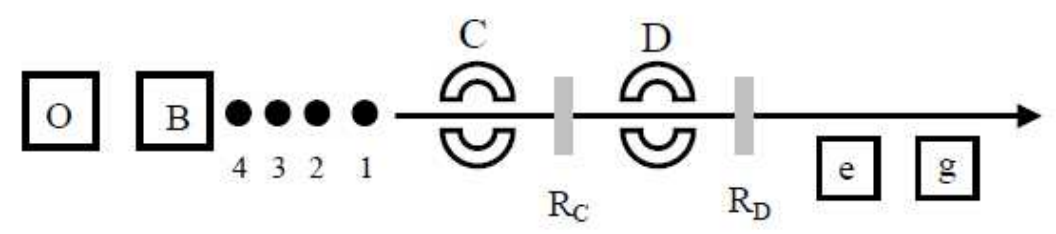

Figure 13. The experimental setup for measuring the concurrence in microwave 3D cavity quantum electrodynamics (QED) using two cavities and two Ramsey regions in [53].

In 2008, Lee et al. also proposed a protocol for directly measuring the concurrence of an arbitrary two-qubit cavity system based on the cavity quantum electrodynamics (QED) technique [54]. The scheme can work for any arbitrary pure state of a two-qubit cavity system, even when the parties do not have knowledge about the state. The scheme derives from the realization that the concurrence coincides with the two-particle visibility under suitable interferometric setups [87-94].

The key element of the protocol is the single-particle interference, whose principle is shown in Figure 14 [95]. The two cavities $\mathrm{A}$ and $\mathrm{B}$ are prepared in the entangled state as $|\varphi\rangle_{1}=\alpha|0\rangle_{A}|1\rangle_{B}+$ $\beta|1\rangle_{A}|0\rangle_{B}$, where $\alpha=|\alpha| e^{i \theta_{a}}, \beta=|\beta| e^{i \theta_{a}}$ and $|\alpha|^{2}+|\beta|^{2}=1 .|1\rangle_{A(B)}$ and $|0\rangle_{A(B)}$ represent the single photon state and vacuum state of cavity A (B), respectively. The party makes a two-level atom in its ground state $|g\rangle$ pass through Cavity A, the dispersive interaction region, and Cavity B, successively. The interaction time between the atom and Cavity $\mathrm{A}$ is chosen corresponding to a $\pi$-pulse interaction. The dispersive interaction shifts the phase of the atomic state by $\Phi$ only under the case that the atom is in the upper state $|e\rangle$. Therefore, it actually acts as a quantum phase gate for the atom [96,97]. The interaction time between the atom and Cavity B is chosen corresponding to a $\pi / 2$-pulse interaction. Thus, after the atom passes through Cavity $\mathrm{A}$, the dispersive interaction region, and Cavity B, successively, the final state of the atom-cavity system can be described as:

$$
|\psi\rangle_{f}=\frac{1}{\sqrt{2}}\left(\alpha-\beta e^{i \Phi}\right)|g\rangle|0\rangle_{A}|1\rangle_{B}-\frac{i}{\sqrt{2}}\left(\alpha+\beta e^{i \Phi}\right)|e\rangle|0\rangle_{A}|0\rangle_{B}
$$

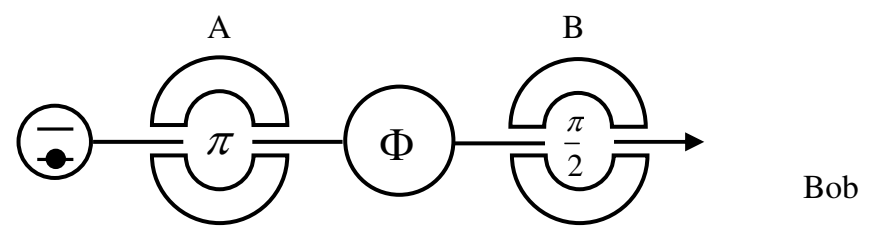

Figure 14. The schematic drawing of the single-photon interference in [54]. A two-level atom is prepared in the ground state $|g\rangle$. The party makes the atom pass through Cavity A, a dispersive interaction region, and Cavity $\mathrm{B}$, successively. The interaction times between the atom and Cavity A, B correspond to the $\pi$-pulse and $\pi / 2$-pulse interactions, respectively. The dispersive interaction can change the relative phase of the atomic states by $\Phi$.

In this way, the probability of obtaining the output atom in the upper state $|e\rangle$ can be written as:

$$
P_{e}=\frac{1}{2}\left[1+2|\alpha \beta| \cos \left(\Phi-\theta_{a}+\theta_{b}\right)\right] .
$$


The probability $P_{e}$ exhibits one-particle interference fringes. There are two possible paths for the atom to end up in $|e\rangle$, that is it can absorb a photon and transmit to $|e\rangle$ in Cavity A or B [95]. The visibility of this interference pattern is $2|\alpha \beta|$, which coincides with the concurrence of the initial cavity state $|\varphi\rangle_{1}$.

The protocol in [54] is suitable for measuring the concurrence of an arbitrary pure entangled state for the two-qubit system as:

$$
|\varphi\rangle_{2}=\alpha|0\rangle_{A}|0\rangle_{B}+\beta|0\rangle_{A}|1\rangle_{B}+\gamma|1\rangle_{A}|0\rangle_{B}+\delta|1\rangle_{A}|1\rangle_{B}
$$

The arbitrary two-qubit cavity state has been successfully generated in [98].

The schematic principle of the protocol is shown in Figure 15. Both Atoms 1 and 2 are prepared in the ground state $|g\rangle_{1}$ and $|g\rangle_{2}$. The parties make each of them pass through the cavity, the dispersive interaction region, and a Ramsey zone, successively. The interaction times between the cavity and the atom are controlled corresponding to the $\pi$-pulse interaction, and the phase shifts generated in the dispersive interaction region are $\Phi_{1}$ and $\Phi_{2}$, respectively. The interaction times in the Ramsey zone $R_{1}$ and $R_{2}$ correspond to the $2 \theta_{1}$ - and $2 \theta_{2}$-pulse, respectively. After the series of interactions, the final state of the two atoms and two cavities can be written as:

$$
|\varphi\rangle_{2 f}=|0\rangle_{A}|0\rangle_{B}\left(A|g\rangle_{1}|g\rangle_{2}+B|g\rangle_{1}|e\rangle_{2}+C|e\rangle_{1}|g\rangle_{2}+D|e\rangle_{1}|e\rangle_{2}\right),
$$

with the coefficients:

$$
\left(\begin{array}{c}
A \\
B \\
C \\
D
\end{array}\right)=M_{1} \otimes M_{2}\left(\begin{array}{c}
\alpha \\
\beta \\
\gamma \\
\delta
\end{array}\right)
$$

The matrix $M_{j}(j=1,2)$ describes the series interaction on the atom $\mathrm{j}$, which can be written as:

$$
M_{j}=\left(\begin{array}{cc}
\cos \theta_{j} & -i \sin \theta_{j} \\
-i \sin \theta_{j} & \cos \theta_{j}
\end{array}\right)\left(\begin{array}{cc}
1 & 0 \\
0 & e^{i \Phi_{j}}
\end{array}\right)\left(\begin{array}{cc}
1 & 0 \\
0 & -i
\end{array}\right) .
$$
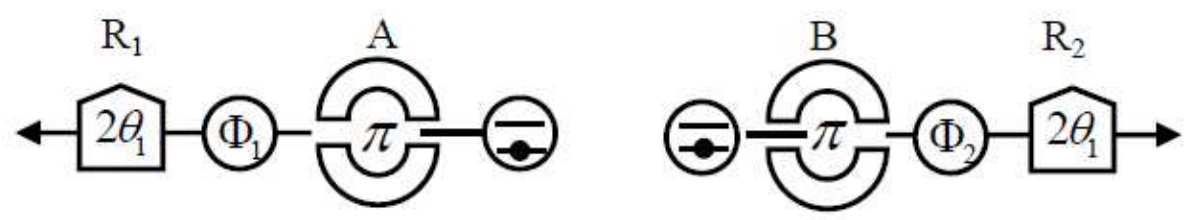

Figure 15. The schematic principle of the protocol in [54]. Both Atoms 1 and 2 are prepared in the ground state $|g\rangle$. The parties make them pass through a cavity, a dispersive interaction region, and a Ramsey zone, successively. The interaction times between the cavity and the atom are chosen to correspond to a $\pi$-pulse interaction. The phase shifts generated in the two dispersive interaction regions are controlled as $\Phi_{1}$ and $\Phi_{2}$, respectively. The interaction times in the two Ramsey zones are chosen to correspond to the phase shifts of $2 \theta_{1}$ and $2 \theta_{2}$, respectively. 
Referring to the investigate results of $[87,88,90]$, we can obtain the corrected joint probability $\bar{P}_{e_{1} e_{2}}$ as:

$$
\begin{aligned}
\bar{P}_{e_{1} e_{2}} & =|D|^{2}-\left(|C|^{2}+|D|^{2}\right)\left(|B|^{2}+|D|^{2}\right)+\frac{1}{4}=|A|^{2}|D|^{2}-|B|^{2}|C|^{2}+\frac{1}{4} \\
& =(|A||D|-|B||C|)(|A||D|+|B||C|)+\frac{1}{4} .
\end{aligned}
$$

Since for arbitrary coefficients A, B, C and D, we can have:

$$
\begin{aligned}
& -|A D-B C| \leq|A||D|-|B||C| \leq|A D-B C| \\
& |A||D|+|B||C| \leq \frac{|A|^{2}+|B|^{2}+|C|^{2}+|D|^{2}}{2}=\frac{1}{2} .
\end{aligned}
$$

we can finally obtain:

$$
\frac{1-2|A D-B C|}{4} \leq \bar{P}_{e_{1} e_{2}} \leq \frac{1+2|A D-B C|}{4} .
$$

After the operations shown in Figure 15, we can obtain:

$$
2|A D-B C|=2|\alpha \delta-\beta \gamma|=C\left(|\varphi\rangle_{2}\right) .
$$

In this way, Equation (69) can be rewritten as:

$$
\frac{1-C\left(|\varphi\rangle_{2}\right)}{4} \leq \bar{P}_{e_{1} e_{2}} \leq \frac{1+C\left(|\varphi\rangle_{2}\right)}{4} .
$$

Hence, the proposed system in Figure 15 provides an effective way to directly measure the concurrence of a two-qubit cavity system.

Recently, Zhou et al. put forward a direct concurrence measurement protocol for nonlocal atomic entanglement assisted by single photons [55]. This protocol is also based on the photonic Faraday rotation in the low-Q cavities. The schematic principle of the photonic Faraday rotation has been briefly explained in Section 2. In the protocol, the parties prepare two identical copies of two-atom entangled states with the form of:

$$
\begin{aligned}
|\Phi\rangle_{a 1 b 1} & =\alpha|0\rangle_{a 1}|0\rangle_{b 1}+\beta|0\rangle_{a 1}|1\rangle_{b 1}+\gamma|1\rangle_{a 1}|0\rangle_{b 1}+\delta|1\rangle_{a 1}|1\rangle_{b 1}, \\
|\Phi\rangle_{a 2 b 2} & =\alpha|0\rangle_{a 2}|0\rangle_{b 2}+\beta|0\rangle_{a 2}|1\rangle_{b 2}+\gamma|1\rangle_{a 2}|0\rangle_{b 2}+\delta|1\rangle_{a 2}|1\rangle_{b 2} .
\end{aligned}
$$

Here, $|0\rangle \equiv\left|g_{L}\right\rangle$ and $|1\rangle \equiv\left|g_{R}\right\rangle$. The two copies of atom states are trapped in the low-Q cavities in the spatial modes of $a_{1}$ and $b_{1}, a_{2}$ and $b_{2}$, respectively.

The schematic drawing of the protocol is shown in Figure 16. The protocol includes two steps. In the first step, the parties prepare two single photons in $\frac{1}{\sqrt{2}}(|L\rangle+|R\rangle)$. They make each of the two photons pass through two cavities, successively. The state of the whole four-atom system can be written as:

$$
\begin{aligned}
& |\Phi\rangle_{a 1 b 1} \otimes|\Phi\rangle_{a 2 b 2} \\
= & \left(\alpha|0\rangle_{a 1}|0\rangle_{b 1}+\beta|0\rangle_{a 1}|1\rangle_{b 1}+\gamma|1\rangle_{a 1}|0\rangle_{b 1}+\delta|1\rangle_{a 1}|1\rangle_{b 1}\right) \\
& \otimes\left(\alpha|0\rangle_{a 2}|0\rangle_{b 2}+\beta|0\rangle_{a 2}|1\rangle_{b 2}+\gamma|1\rangle_{a 2}|0\rangle_{b 2}+\delta|1\rangle_{a 2}|1\rangle_{b 2}\right) \\
= & \alpha^{2}|0\rangle_{a 1}|0\rangle_{a 2}|0\rangle_{b 1}|0\rangle_{b 2}+\alpha \beta|0\rangle_{a 1}|0\rangle_{a 2}|0\rangle_{b 1}|1\rangle_{b 2}+\alpha \gamma|0\rangle_{a 1}|1\rangle_{a 2}|0\rangle_{b 1}|0\rangle_{b 2}+\alpha \delta|0\rangle_{a 1}|1\rangle_{a 2}|0\rangle_{b 1}|1\rangle_{b 2} \\
& +\alpha \beta|0\rangle_{a 1}|0\rangle_{a 2}|1\rangle_{b 1}|0\rangle_{b 2}+\beta^{2}|0\rangle_{a 1}|0\rangle_{a 2}|1\rangle_{b 1}|1\rangle_{b 2}+\beta \gamma|0\rangle_{a 1}|1\rangle_{a 2}|1\rangle_{b 1}|0\rangle_{b 2}+\beta \delta|0\rangle_{a 1}|1\rangle_{a 2}|1\rangle_{b 1}|1\rangle_{b 2} \\
& +\alpha \gamma|1\rangle_{a 1}|0\rangle_{a 2}|0\rangle_{b 1}|0\rangle_{b 2}+\beta \gamma|1\rangle_{a 1}|0\rangle_{a 2}|0\rangle_{b 1}|1\rangle_{b 2}+\gamma^{2}|1\rangle_{a 1}|1\rangle_{a 2}|0\rangle_{b 1}|0\rangle_{b 2}+\gamma \delta|1\rangle_{a 1}|1\rangle_{a 2}|0\rangle_{b 1}|1\rangle_{b 2} \\
& +\alpha \delta|1\rangle_{a 1}|0\rangle_{a 2}|1\rangle_{b 1}|0\rangle_{b 2}+\beta \delta|1\rangle_{a 1}|0\rangle_{a 2}|1\rangle_{b 1}|1\rangle_{b 2}+\gamma \delta|1\rangle_{a 1}|1\rangle_{a 2}|1\rangle_{b 1}|0\rangle_{b 2}+\delta^{2}|1\rangle_{a 1}|1\rangle_{a 2}|1\rangle_{b 1}|1\rangle_{b 2} .
\end{aligned}
$$


After passing through the cavities, if both photons do not change their polarization, they will transmit the polarization beam splitters (PBSs) and finally be detected by single-photon detectors $D_{1}$ and $D_{3}$. The PBS can transmit the photon in $|+\rangle=\frac{1}{\sqrt{2}}(|L\rangle+|R\rangle)$, while reflecting the photon in $|-\rangle=\frac{1}{\sqrt{2}}(|L\rangle-$ $|R\rangle)$. Under the case that both photons do not change their polarization, the whole four-atom state in Equation (74) will collapse to:

$$
\begin{aligned}
|\Phi\rangle_{a 1 a 2 b 1 b 2}= & \frac{\alpha \delta}{\sqrt{2\left(|\alpha \delta|^{2}+|\beta \gamma|^{2}\right)}}\left(|0\rangle_{a 1}|1\rangle_{a 2}|0\rangle_{b 1}|1\rangle_{b 2}+|1\rangle_{a 1}|0\rangle_{a 2}|1\rangle_{b 1}|0\rangle_{b 2}\right) \\
& +\frac{\beta \gamma}{\sqrt{2\left(|\alpha \delta|^{2}+|\beta \gamma|^{2}\right)}}\left(|0\rangle_{a 1}|1\rangle_{a 2}|1\rangle_{b 1}|0\rangle_{b 2}+|1\rangle_{a 1}|0\rangle_{a 2}|0\rangle_{b 1}|1\rangle_{b 2}\right),
\end{aligned}
$$

with the probability of $P_{1}=2|\alpha \delta|^{2}+2|\beta \gamma|^{2}$.

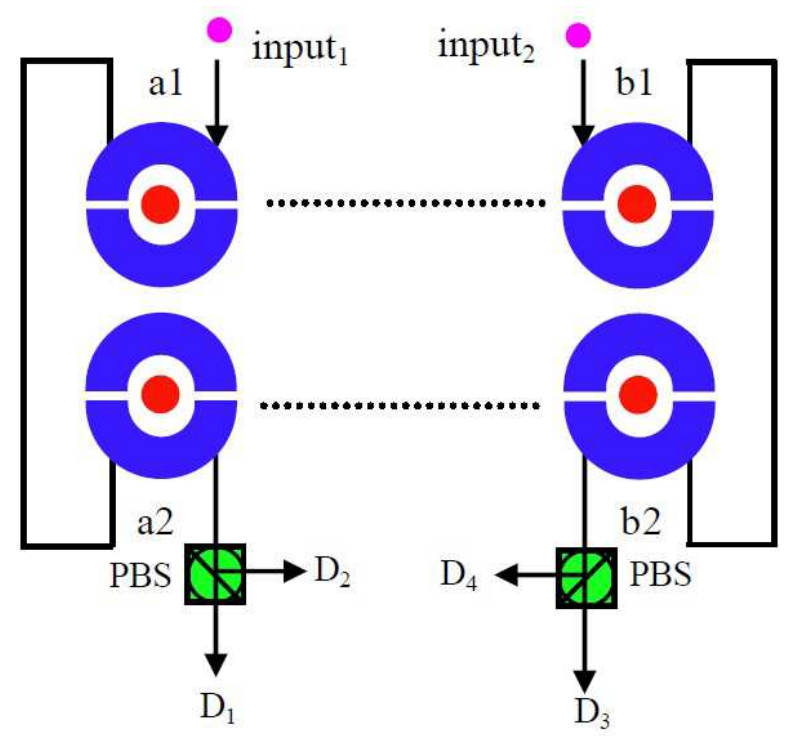

Figure 16. The schematic principle for directly measuring the two-atom entangled state in [55]. The parties make each of two input single photons with the form of $\frac{1}{\sqrt{2}}(|L\rangle+|R\rangle)$ pass through two low-Q cavities, successively. The polarization beam splitter (PBS) can transmit the photon in the $|+\rangle$ polarization and reflect the photon in the $|-\rangle$ polarization. Here, $| \pm\rangle$ represent $\frac{1}{\sqrt{2}}(|L\rangle \pm|R\rangle)$. The output photons from the PBS will be detected by the single photon detectors $D_{1}, D_{2}, D_{3}$ or $D_{4}$.

Then, the parties perform the Hadamard operations on atoms $a_{1}$ and $a_{2}$ by driving the atoms with an external classical field (polarized lasers), which makes $|\Phi\rangle_{a 1 a 2 b 1 b 2}$ evolve to:

$$
\begin{aligned}
|\Phi\rangle_{a 1 a 2 b 1 b 2}^{\prime}= & \frac{\alpha \delta+\beta \gamma}{2 \sqrt{2\left(|\alpha \delta|^{2}+|\beta \gamma|^{2}\right)}}\left(|0\rangle_{a 1}|0\rangle_{a 2}-|1\rangle_{a 1}|1\rangle_{a 2}\right) \otimes\left(|0\rangle_{b 1}|1\rangle_{b 2}+|1\rangle_{b 1}|0\rangle_{b 2}\right) \\
& +\frac{\alpha \delta-\beta \gamma}{2 \sqrt{2\left(|\alpha \delta|^{2}+|\beta \gamma|^{2}\right)}}\left(|0\rangle_{a 1}|1\rangle_{a 2}-|1\rangle_{a 1}|0\rangle_{a 2}\right) \otimes\left(|0\rangle_{b 1}|1\rangle_{b 2}-|1\rangle_{b 1}|0\rangle_{b 2}\right) .
\end{aligned}
$$

In the second step, the parties prepare another auxiliary single photon in $\frac{1}{\sqrt{2}}(|L\rangle+|R\rangle)$ in the Input 1 mode and make it pass through the cavities $a_{1}$ and $a_{2}$, successively. After the cavities, if the single 
photon does not change its polarization and is finally detected by $D_{1}$ for the second time, $|\Phi\rangle_{a 1 a 2 b 1 b 2}^{\prime}$ will finally collapse to:

$$
|\Phi\rangle_{a 1 a 2 b 1 b 2}^{\prime \prime}=\frac{1}{2}\left(|0\rangle_{a 1}|1\rangle_{a 2}-|1\rangle_{a 1}|0\rangle_{a 2}\right) \otimes\left(|0\rangle_{b 1}|1\rangle_{b 2}-|1\rangle_{b 1}|0\rangle_{b 2}\right)
$$

with the success probability of $P_{2}=\frac{|\alpha \delta-\beta \gamma|^{2}}{2\left(|\alpha \delta|^{2}+|\beta \gamma|^{2}\right)}$.

So far, the protocol is completed. The total success probability of obtaining $|\Phi\rangle_{a 1 a 2 b 1 b 2}^{\prime \prime}$ is:

$$
P=P_{1} P_{2}=|\alpha \delta-\beta \gamma|^{2}=\frac{C^{2}(\Phi)}{4} .
$$

In this way, the aim of measuring nonlocal atomic entanglement can be transformed to measure the success probability $\mathrm{P}$ of the state $|\Phi\rangle_{a 1 a 2 b 1 b 2}^{\prime \prime}$.

\section{Discussion and Conclusion}

Thus far, we have briefly explained some direct concurrence measurement protocols for the optical states and atomic states. Different from traditional concurrence measurement protocols, these protocols encode the concurrence into the success probability for picking up the balanced state. Moreover, the measurement technique not only can deal with the pure state, but also can deal with the mixed state. The key elements of these protocols are the parity check gates, which can be constructed by the linear optical elements, such as PBS, HWP and BS, or the nonlinear optical elements, such as the cross-Kerr material and the cavity. The setups constructed by the linear optical elements can be experimental realized under the current experimental condition. On the other hand, the realization of nonlinear optical elements has been a challenge for a long time. Therefore, in the paper, we especially discuss the parity check gate constructed by the cross-Kerr nonlinearity and the QED in the low-Q cavity.

In Figures 3, 4, 13 and 15, the cross-Kerr nonlinearities are used to make the parity check for the spatial degree of freedom, while in Figures 5 and 14, the cross-Kerr nonlinearities combined with the PBSs are adopted to make the parity check for the polarization degree of freedom. In Figures 3-5, 13 and 14, two kinds of Kerr materials are required. One is to generate the phase shift of $\theta$, and the other is to generate $-\theta$. Meanwhile, we need to make the $\pm \theta$ undistinguishable in the homodyne measurement. On the other hand, in Figure 15, four kinds of Kerr materials are required, corresponding to the phase shift of $\theta_{1}, \theta_{2}, \theta_{3}$ and $\theta_{4}$, respectively. Although the cross-Kerr nonlinearity has been widely discussed in current quantum information processing [99-104]. The cross-Kerr nonlinearity has been regarded as a controversial topic in practical experiments for a long time [105-107]. There are two main reasons. First, during the homodyne detection process, the qubit states may degrade to the mixed states [108-112]. Second, the natural cross-Kerr nonlinearity is extremely weak, which makes it difficult to discriminate two overlapping coherent states in homodyne detection. For example, as pointed out by Kok et al., in the optical single-photon regime, the Kerr phase shift is only about $10^{-18}$. In this way, Shapiro once showed that Kerr nonlinearity at the single-photon level cannot contribute to benefiting quantum computation [106,107]. On the other hand, in current technology, it is quite a controversial assumption to obtain a clean cross-Kerr nonlinearity [113,114]. Fortunately, according to [108], we can make the decoherence extremely weak, simply by an arbitrary strong coherent state associated with a displacement $\mathrm{D}(-\alpha)$ performed on the coherent state. Meanwhile, with the help of weak measurement, it is possible 
to obtain an observable cross-Kerr phase shift with amplification [115]. Hofmann showed that with the help of a single two-level atom trapped in a one-side cavity, we can obtain a phase shift as large as $\pi$ [116]. As shown in [117], large cross-Kerr nonlinearities were also obtained in a double-quantum-well structure with a four-level, double-type configuration. The "giant" cross-Kerr effect with a phase shift of 20 degrees per photon has been observed in the current experiment [118]. Recent work also showed that the Rydberg atom system can generate a rather large cross-phase between photons [119]. Therefore, recent theoretical and experimental works based on cross-Kerr nonlinearity may provide its practical application in future quantum information processing.

Then, we provide a discussion of the QED in the optical microcavity. In Figures 7, 8 and 10, we adopt the photonic Faraday rotation to realize the parity check measurement, which has been quite popular in recent years. In Figures 7 and 8, the parties encode a three-level atom in the low-Q cavity and make a photon pass through the cavity to interact with the three-level atom. In Figure 10, we make a single-photon entanglement state encode in two cavities and make an atom pass through the cavities and interact with the photon state. In both protocols, we can measure the concurrence based on the input-output processing. It is worth mentioning that these cavity QED steps to measure the concurrence are analogous to the steps for engineering and measuring generalized binomial states of radiation [120-125]. Recently, some works about the realization of the photonic Faraday rotation have been reported. For example, certain studies have reported on ${ }^{85} \mathrm{Rb}$ coupled to an optical cavity. In 2005, $\mathrm{Nu} \beta$ ann et al. reported that they could precisely control and adjust the individual ultracold ${ }^{85} \mathrm{Rb}$ atoms coupled to a high-finesse optical cavity [126]. In 2007, Fortier et al. reported that by incorporating a deterministically loaded atom conveyor, they could realize methods for deterministic loading of single

${ }^{85} \mathrm{Rb}$ atoms into a cavity [127]. In the same year, the group of Colombe reported that they could realize strong atom-field coupling for Bose-Einstein condensates in a Fabry-Perot cavity on a chip [128].

\section{Acknowledgments}

This work is supported by the National Natural Science Foundation of China under Grant Nos. 11474168 and 61401222, the Qing Lan Project in Jiangsu Province and the Priority Academic Program Development of Jiangsu Higher Education Institutions.

\section{Author Contributions}

In the paper, Yu-Bo Sheng collects the related literatures and draws the figures, while Lan Zhou writes the manuscript. All authors have read and approved the final manuscript.

\section{Conflicts of Interest}

The authors declare no conflict of interest.

\section{References}

1. Einstein, A.; Podolsky, B.; Rosen, N. Can quantum-mechanical description of physical reality be considered complete? Phys. Rev. 1935, 47, 777. 
2. Schrödinger, E. Die gegenwärtige situation in der quantenmechanik. Naturwissenschaften 1935, 23, 823-828.

3. Nielsen, M.A.; Chuang, I.L. Quantum Computation and Quantum Information; Cambridge University Press: Cambridge, UK, 2000.

4. Bennett, C.H.; Brassard, G.; Crepeau, C.; Jozsa, R.; Peres, A.; Wootters, W.K. Teleporting an unknown quantum state via dual classical and Einstein-Podolsky-Rosen channels. Phys. Rev. Lett. 1993, 70, 1895-1899.

5. Bouwmeester, D.; Pan, J.W.; Mattle, K.; Eibl, M.; Weinfurter, H.; Zeilinger, A. Experimental quantum teleportation. Nature 1997, 390, 575-579.

6. Bennett, C.H.; DiVincenzo, D.P. Quantum information and computation. Nature 2000, 404, 247-255.

7. Marzolino, U.; Buchleitner, A. Quantum teleportation with identical particles. Phys. Rev. A 2015, 91, 032316.

8. Liu, X.S.; Long, G.L.; Tong, D.M.; Li, F. General scheme for superdense coding between multi-parties. Phys. Rev. A 2002, 65, 022304.

9. Karlsson, A.; Bourennane, M. Superdense coding of quantum states. Phys. Rev. Lett. 2004, 92, 187901.

10. Ekert, A.K. Quantum cryptography based on Bell's theorem. Phys. Rev. Lett. 1991, 67, 661-663.

11. Zhang, C.M.; Song, X.T.; Treeviriyanupab, P.; Li, M.; Wang, C.; Li, H.W.; Yin, Z.Q.; Chen, W.; Han, Z.F. Delayed error verification in quantum key distribution. Chin. Sci. Bull. 2014, 59, 2825-2828.

12. Su, X.L. Applying Gaussian quantum discord to quantum key distribution. Chin. Sci. Bull. 2014, 59, 1083-1090.

13. Long, G.L.; Liu, X.S. Theoretically efficient high-capacity quantum-key-distribution scheme. Phys. Rev. A 2002, 65, 032302.

14. Deng, F.G.; Long, G.L.; Liu, X.S. Two-step quantum direct communication protocol using the Einstein-Podolsky-Rosen pair block. Phys. Rev. A 2003, 68, 042317.

15. Chang, Y.; Xu, C.X.; Zhang, S.B.; Yan, L. Quantum secure direct communication and authentication protocol with single photons. Chin. Sci. Bull. 2013, 58, 4571-4576.

16. Wei, H.R.; Deng, F.G. Compact quantum gates on electron-spin qubits assisted by diamond nitrogen-vacancy centers inside cavities. Phys. Rev. A 2013, 88, 042323.

17. Wei, H.R.; Deng, F.G. Universal quantum gates on electron-spin qubits with quantum dots inside single-side optical microcavities. Opt. Express 2014, 22, 593-607.

18. Ren, B.C.; Deng, F.G. Hyper-parallel photonic quantum computation with coupled quantum dots. Sci. Rep. 2014, 4, 4623.

19. Ren, B.C.; Wang, G.Y.; Deng, F.G. Universal hyperparallel hybrid photonic quantum gates with dipole-induced transparency in the weak-coupling regime. Phys. Rev. A 2015, 91, 032328.

20. Liu, Y. Deleting a marked state in quantum database in a duality computing mode. Chin. Sci. Bull. 2013, 58, 2927-2931.

21. Liu, Y.; Ou-Yang, X.P. A quantum algorithm that deletes marked states from an arbitrary database. Chin. Sci. Bull. 2013, 58, 2329-2333. 
22. Zheng, C.; Long, G.F. Quantum secure direct dialogue using Einstein-Podolsky-Rosen pairs. Sci. Chin. Phys. Mech. Astron. 2014, 57, 1238-1243.

23. Su, X.L.; Jia, X.J.; Xie, C.D.; Peng, K.C. Preparation of multipartite entangled states used for quantum information networks. Sci. Chin. Phys. Mech. Astron. 2014, 57, 1210-1217.

24. Leibfried, D.; Knill, E.; Seidelin, S.; Britton, J.; Blakestad, R.B.; Chiaverini, J.; Hume, D.B.; Itano, W.M.; Jost, J.D.; Langer, C.; et al. Creation of a six-atom 'Schrödinger cat' state. Nature 2005, 438, 639-642.

25. Häffner, H.; Hänsel, W.; Roos, C.F.; Benhelm, J.; Chek-al-kar, D.; Chwalla, M.; Körber, T.; Rapol, U.D.; Riebe, M.; Schmidt, P.O.; Becher, C.; Gühne, O.; Dür, W.; Blatt, R. Scalable multiparticle entanglement of trapped ions. Nature 2005, 438, 643-646.

26. Lu, C.Y.; Zhou, X.Q.; Gühne, O.; Gao, W.B.; Zhang, J.; Yuan, Z.S.; Goebel, A.; Yang, T.; Pan, J.W. Experimental entanglement of six photons in graph states. Nat. Phys. 2007, 3, 91-95.

27. Gao, W.B.; Lu, C.Y.; Yao, X.C.; Xu, P.; Gühne, O.; Goebel, A.; Chen, Y.A.; Peng, C.Z.; Chen, Z.B.; Pan, J.W. Experimental demonstration of a hyper-entangled ten-qubit Schrödinger cat state. Nat. Phys. 2010, 6, 331-335.

28. Hald, J.; Sörensen, J.L.; Schori, C.; Polzik, E.S. Spin squeezed atoms: A macroscopic entangled ensemble created by light. Phys. Rev. Lett. 1999, 83, 1319-1322.

29. Mandel, O.; Greiner, M.; Widera, A.; Rom, T.; Hänsch, T.; Bloch, I. Controlled collisions for multi-particle entanglement of optically trapped atoms. Nature 2003, 425, 937-940.

30. Huang, Y.F.; Liu, B.H.; Peng, L.; Li, Y.H.; Li, L.; Li, C.F.; Guo, G.C. Experimental generation of an eight-photon Greenberger-Horne-Zeilinger state. Nat. Commun. 2011, 2, 546.

31. Yao, X.C.; Wang, T.X.; Xu, P.; Lu, H.; Pan, G.S.; Bao, X.H.; Peng, C.Z.; Lu, C.Y.; Chen, Y.A.; Pan, J.W. Observation of eight-photon entanglement. Nat. Photon. 2012, 6, 225-228.

32. He, L.; Chen, L.K.; Liu, C.; Xu, P.; Yao, X.C.; Li, L.; Liu, N.L.; Zhao, B.; Chen, Y.A.; Pan, J.W. Experimental realization of a concatenated Greenberger-Horne-Zeilinger state for macroscopic quantum superpositions. Nat. Photon. 2014, 8, 364-368.

33. Bell, J.S. On the Einstein-Podolsky-Rosen paradox. Physics 1964, 1, 195-200.

34. Horodecki, M.; Horodecki, P.; Horodecki, R. Separability of mixed states: Necessary and sufficient conditions. Phys. Lett. A 1996, 223, 1-8.

35. Bartkiewicz, K.; Beran, J.; Lemr, K.; Norek, M.; Miranowicz, A. Quantifying entanglement of a two-qubit system via measurable and invariant moments of its partially transposed density matrix. Phys. Rev. A 2015, 91, 022323.

36. Bartkiewicz, K.; Horodecki, P.; Lemr, K.; Miranowicz, A.; Życzkowski, K. Method for universal detection of two-photon polarization entanglement. Phys. Rev. A 2015, 91, 032315.

37. Gühne, O.; Tóth, G. Entanglement detection. Phys. Rep. 2009, 474, 1-75.

38. James, D.F.V.; Kwiat, P.G.; Munro, W.J.; White, A.G. Measurement of qubits. Phys. Rev. A 2005, $64,052312$.

39. Mohammadi, M.; Brańczyk, A.M.; James, D.F.V. Fourier-transform quantum state tomography. Phys. Rev. A 2013, 87, 012117.

40. White, A.G.; James, D.F.V.; Eberhard, P.H.; Kwiat, P.G. Nonmaximally entangled states: Production, characterization, and utilization. Phys. Rev. Lett. 1999, 83, 3103-3107. 
41. Bennett, C.H.; Bernstein, H.J.; Popescu, S.; Schumacher, B. Concentrating partial entanglement by local operations. Phys. Rev. A 1996, 53, 2046-2052.

42. Bennett, C.H.; Divincenze, D.P.; Smolin, J.A.; Wootters, W.K. Mixed-state entanglement and quantum error correction. Phys. Rev. A 1996, 54, 3824-3851.

43. Wootters, W.K. Entanglement of formation and concurrence. Quantum Inf. Comput. 2001, 1, $27-44$.

44. Hill, S.; Wootters, W.K. Entanglement of a pair of quantum bits. Phys. Rev. Lett. 1997, 78, 5022-5025.

45. Wootters, W.K. Entanglement of formation of an arbitrary state of two qubits. Phys. Rev. Lett. 1998, 80, 2245-2248.

46. Mintert, F.; KuŚ, M.; Buchleitner, A. Concurrence of mixed multipartite quantum states. Phys. Rev. Lett. 2005, 95, 260502.

47. Fei, S.M.; Zhao M.J.; Chen, K.; Wang, Z.X. Experimental determination of entanglement for arbitrary pure states. Phys. Rev. A 2009, 80, 032320.

48. Walborn, S.P.; Souto Ribeiro, P.H.; Davidovich, L.; Mintert, F.; Buchleitner, A. Experimental determination of entanglement with a single measurement. Nature 2006, 440, 1022-1024.

49. Walborn, S.P.; Souto Ribeiro, P.H.; Davidovich, L.; Mintert, F.; Buchleitner, A. Experimental determination of entanglement by a projective measurement. Phys. Rev. A 2007, 75, 032338.

50. Zhang, L.H.; Yang, Q.; Yang, M.; Song, W.; Cao, Z.L. Direct measurement of the concurrence of two-photon polarization-entangled states. Phys. Rev. A 2013, 88, 062342.

51. Zhang, L.H.; Yang, M.; Cao, Z.L. Direct measurement of the concurrence for two-photon polarization entangled pure states by parity-check measurements. Phys. Lett. A 2013, 377, 1421-1424.

52. Zhou, L. Measurement of arbitrary two-photon entanglement state with the photonic Faraday rotation. 2014, arXiv:1401.6719.

53. Romero, G.; López, C.E.; Lastra, F.; Solano, E.; Retamal, J.C. Direct measurement of concurrence for atomic two-qubit pure states. Phys. Rev. A 2007, 75, 032303.

54. Lee, S.M.; Ji, S.W.; Lee, H.W.; Zubairy, M.S. Proposal for direct measurement of concurrence via visibility in a cavity QED system. Phys. Rev. A 2008, 77, 040301(R).

55. Zhou, L.; Sheng, Y.B. Detection of nonlocal atomic entanglement assisted by single photons. Phys. Rev. A 2014, 90, 024301.

56. Liu, J.; Zhou, L.; Sheng, Y.B. Direct measurement of the concurrence for two-qubit electron spin entangled pure state base on charge detection. Chin. Phys. B 2015, 24, 070309.

57. Sheng, Y.B.; Guo, R.; Pan, J.; Zhou, L.; Wang, X.F. Two-step measurement of the concurrence for hyperentangled state. Quantum Inf. Process. 2015, 14, 963-978.

58. Kwiat, P.G. Hyper entangled states. J. Mod. Opt. 1997, 44, 2173-2184.

59. Barbieri, M.; Cinelli, C.; Mataloni, P.; Martini, F.D. Polarization-momentum hyperentangled states: Realization and characterization. Phys. Rev. A 2005, 72, 052110.

60. Rarity, J.; Tapster, P. Experimental violation of Bell's inequality based on phase and momentum. Phys. Rev. Lett. 1990, 64, 2495-2498. 
61. Kwiat, P.G.; Waks, E.; White, A.G.; Appelbaum, I.; Eberhard, P.H. Ultrabright source of polarization-entangled photons. Phys. Rev. A 1999, 60, R773-R776.

62. Fiorentino, M.; Wong, F.N.C. Deterministic controlled-not gate for single photon two-qubit quantum logic. Phys. Rev. Lett. 2004, 93, 070502.

63. Sheng, Y.B.; Zhou, L.; Zhao, S.M.; Zheng, B.Y. Efficient single-photon-assisted entanglement concentration for partially entangled photon pairs. Phys. Rev. A 2012, 85, 012307.

64. Sheng, Y.B.; Zhou, L.; Zhao, S.M. Efficient two-step entanglement concentration for arbitrary W states. Phys. Rev. A 2012, 85, 042302.

65. Sheng, Y.B.; Zhou, L. Deterministic entanglement distillation for secure double-server blind quantum computation. Sci. Rep. 2015, 5, 7815.

66. Zhou, L.; Sheng, Y.B.; Cheng, W.W.; Gong, L.Y.; Zhao, S.M. Efficient entanglement concentration for arbitrary less-entangled NOON states. Quantum Inf. Process. 2013, 12, 1307-1320.

67. Zhou, L.; Sheng, Y.B.; Cheng, W.W.; Gong, L.Y.; Zhao, S.M. Efficient entanglement concentration for arbitrary single-photon multimode W state. J. Opt. Soc. Am. B 2013 30, $71-77$.

68. Zhou, L.; Sheng, Y.B. Efficient single-photon entanglement concentration for quantum communications. Opt. Commun. 2014, 313, 217-222.

69. Zhou, L.; Sheng, Y.B. Recyclable amplification protocol for the single-photon entangled state. Laser Phys. Lett. 2015, 12, 045203.

70. Song, W.; Yang, M.; Cao, Z.L. Purifying entanglement of noisy two-qubit states via entanglement swapping. Phys. Rev. A 2014, 89, 014303.

71. Guo, Q.; Bai, J.; Cheng, L.Y.; Shao, X.Q.; Wang, H.F.; Zhang, S. Simplified optical quantum-information processing via weak cross-Kerr nonlinearities. Phys. Rev. A 2011, 83, 054303.

72. Nemoto, K.; Munro, W.J. Nearly deterministic linear optical controlled-not gate. Phys. Rev. Lett. 2004, 93, 250502.

73. Wei, T.C.; Altepeter, J.B.; Branning, D.; Goldbart, P.M.; James, D.F.V.; Jeffrey, E.; Kwiat, P.G.; Mukhopadhyay, S.; Peters, N.A. Synthesizing arbitrary two-photon polarization mixed states. Phys. Rev. A 2005, 71, 032329.

74. Collins, D.; Gisin, N. A relevant two qubit Bell inequality inequivalent to the CHSH inequality. J. Phys. A 2004, 37, 1775-1787.

75. An, J.H.; Feng, M.; Oh, C.H. Fidelity in topological quantum phases of matter. Phys. Rev. A 2009, 79, 032303.

76. Chen, J.J.; An, J.H.; Feng, M.; Liu, G. Teleportation of an arbitrary multipartite state via photonic Faraday rotation. J. Phys. B 2010, 43, 095505.

77. Bastos, W.P.; Cardoso, W.B.; Avelar, A.T.; de Almeida, N.G.; Baseia, B. Controlled teleportation via photonic Faraday rotations in low-Q cavities. Quantum Inf. Process. 2012, 11, 1867-1881.

78. Julsgaard, B.; Kozhekin, A.; Polzik, E.S. Experimental long-lived entanglement of two macroscopic objects. Nature 2001, 413, 400-403. 
79. Peng, Z.H.; Zou, J.; Liu, X.J.; Xiao, Y.J.; Kuang, L.M. Atomic and photonic entanglement concentration via photonic Faraday rotation. Phys. Rev. A 2012, 86, 034305.

80. Zhou, L.; Sheng, Y.B. Arbitrary atomic cluster state concentration for one-way quantum computation. J. Opt. Soc. Am. B 2014, 31, 1-10.

81. Zhou, L.; Wang, X.F.; Sheng, Y.B. Efficient entanglement concentration for arbitrary less-entangled N-atom GHZ state. Int. J. Theor. Phys. 2014, 53, 1752-1766.

82. Vallone, G.; Ceccarelli, R.; De Martini, F.; Mataloni, P. Hyperentanglement of two photons in three degrees of freedom. Phys. Rev. A 2009, 79, 030301(R).

83. Horodecki, R.; Horodecki, P.; Horodecki, M.; Horodecki, K. Quantum entanglement. Rev. Mod. Phys. 2009, 81, 865-942.

84. Osnaghi, S.; Bertet, P.; Auffeves, A.; Maioli, P.; Brune, M.; Raimond, J.M.; Haroche, S. Coherent control of an atomic collision in a cavity. Phys. Rev. Lett. 2001, 87, 037902.

85. Zheng, S.B.; Guo, G.C. Efficient scheme for two-atom entanglement and quantum information processing in cavity QED. Phys. Rev. Lett. 2000, 85, 2392-2395.

86. Rauschenbeutel, A.; Nogues, G.; Osnaghi, S.; Bertet, P.; Brune, M.; Raimond, J.M.; Haroche, S. Coherent operation of a tunable quantum phase gate in cavity QED. Phys. Rev. Lett. 2000, 83, $5166-5169$.

87. Jaeger, G.; Horne, M.A.; Shimony, A. Complementarity of one-particle and two-particle interference. Phys. Rev. A 1993, 48, 1023-1027.

88. Jaeger, G.; Shimony, A.; Vaidman, L. Two interferometric complementarities. Phys. Rev. A 1995, 51, 54-67.

89. Horne, M.A.; Shimony, A.; Zeilinger, A. Two-particle interferometry. Phys. Rev. Lett. 1989, 62, 2209-2212.

90. Abouraddy, A.F.; Saleh, B.E.A.; Sergienko, A.V.; Teich, M.C. Degree of entanglement for two qubits. Phys. Rev. A 2001, 64, 050101(R).

91. Kaszlikowski, D.; Kwek, L.C.; Zukowski, M.; Englert, B.G. Information-theoretic approach to single-particle and two-particle interference in multipath interferometers. Phys. Rev. Lett. 2003, 91, 037901.

92. Jakob, M.; Bergou, J. Quantitative conditional quantum erasure in two-atom resonance fluorescence. Phys. Rev. A 2002, 66, 062107.

93. Jakob, M.; Bergou, J.A. Generalized complementarity relations in composite quantum systems of arbitrary dimensions. Int. J. Mod. Phys. B 2006, 20, 1371-1381.

94. de Melo, F.; Walborn, S.P.; Bergou, J.A.; Davidovich, L. Quantum nondemolition circuit for testing bipartite complementarity. Phys. Rev. Lett. 2007, 98, 250501.

95. Zubairy, M.S.; Agarwal, G.S.; Scully, M.O. Quantum disentanglement eraser: A cavity QED implementation. Phys. Rev. A 2004, 70, 012316.

96. Rauschenbeutel, A.; Nogues, G.; Osnaghi, S.; Bertet, P.; Brune, M.; Raimond, J.M.; Haroche, S. Coherent operation of a tunable quantum phase gate in cavity QED. Phys. Rev. Lett. 1999, 83, 5166-5169. 
97. Bertet, P.; Osnaghi, S.; Rauschenbeutel, A.; Nogues, G.; Auffeves, A.; Brune, M.; Raimond, J.M.; Haroche, S. A complementarity experiment with an interferometer at the quantum classical boundary. Nature 2001, 411, 166-170.

98. Di, T.; Zubairy, M.S. Generation of arbitrary two-qubit entangled states in cavity QED. J. Mod. Opt. 2004, 51, 2387-2393.

99. Barrett, S.D.; Kok, P.; Nemoto, K.; Beausoleil, R.G.; Munro, W.J.; Spiller, T.P. Symmetry analyzer for nondestructive Bell-state detection using weak nonlinearities. Phys. Rev. A 2005, 71, 060302(R).

100. He, B.; Lin, Q.; Simon, C. Cross-Kerr nonlinearity between continuous-mode coherent states and single photons. Phys. Rev. A 2011, 83, 053826.

101. He, B.; Scherer, A. Continuous-mode effects and photon-photon phase gate performance. Phys. Rev. A 2012, 85, 033814.

102. He, B.; Ren, Y.; Bergou, J.A. Creation of high-quality long-distance entanglement with flexible resources. Phys. Rev. A 2009, 79, 052323.

103. Lin, Q.; Li, J. Quantum control gates with weak cross-Kerr nonlinearity. Phys. Rev. A 2009, 79, 022301.

104. Lin, Q.; He, B. Single-photon logic gates using minimal resources. Phys. Rev. A 2009, 80, 042310 .

105. Gea-Banacloche, J. Impossibility of large phase shifts via the giant Kerr effect with single-photon wave packets. Phys. Rev. A 2010, 81, 043823.

106. Shapiro, J.H. Single-photon Kerr nonlinearities do not help quantum computation. Phys. Rev. A 2006, 73, 062305.

107. Shapiro, J.H.; Razavi, M. Continuous-time cross-phase modulation and quantum computation. New J. Phys. 2007 , 9, 16.

108. Jeong, H. Quantum computation using weak nonlinearities: Robustness against decoherence. Phys. Rev. A 2006, 73, 052320.

109. Barrett, S.D.; Milburn, G.J. Quantum-information processing via a lossy bus. Phys. Rev. A 2006, 74, 060302(R).

110. Jeong, H.; Kim, M.S.; Ralph, T.C.; Ham B.S. Generation of macroscopic superposition states with small nonlinearity. Phys. Rev. A 2004, 70, 061801(R).

111. Jeong, H. Using weak nonlinearity under decoherence for macroscopic entanglement generation and quantum computation. Phys. Rev. A 2005, 72, 034305.

112. Jeong, H.; An, N.B. Greenberger-Horne-Zeilinger-type and W-type entangled coherent states: Generation and Bell-type inequality tests without photon counting. Phys. Rev. A 2006, 74, 022104.

113. Kok, P.; Munro, W.J.; Nemoto, K.; Ralph, T.C.; Dowling, J.P.; Milburn, G.J. Linear optical quantum computing with photonic qubits. Rev. Mod. Phys. 2007, 79, 135-174.

114. Kok, P.; Lee, H.; Dowling, J.P. Single-photon quantum-nondemolition detectors constructed with linear optics and projective measurements. Phys. Rev. A 2002, 66, 063814.

115. Feizpour, A.; Xing, X.; Steinberg, A.M. Amplifying single-photon nonlinearity using weak measurements. Phys. Rev. Lett. 2011, 107, 133603. 
116. Hofmann, H.F.; Kojima, K.; Takeuchi, S.; Sasaki, K. Optimized phase switching using a single-atom nonlinearity. J. Opt. B 2003, 5, 218-221.

117. Zhu, C.; Huang, G. Giant Kerr nonlinearity, controlled entangled photons and polarization phase gates in coupled quantum-well structures. Opt. Express 2011, 19, 23364-23376.

118. Hoi, I.C.; Kockum, A.F.; Palomaki, T.; Stace, T.M.; Fan, B.; Tornberg, L.; Sathyamoorthy, S.R.; Johansson, G.; Delsing, P.; Wilson, C.M. Giant cross-Kerr effect for propagating microwaves induced by an artificial atom. Phys. Rev. Lett. 2013, 111, 053601.

119. He, B.; Sharypov, A.V.; Sheng, J.; Simon, C.; Xiao, M. Two-photon dynamics in coherent Rydberg atomic ensemble. Phys. Rev. Lett. 2014, 112, 133606.

120. Stoler, D.; Saleh, B.E.A.; Teich, M.C. Binomial states of the quantized radiation field. Optica Acta 1985, 32, 345-355.

121. Vidiella-Barranco A.; Roversi, J.A. Statistical and phase properties of the binomial states of the electromagnetic field. Phys. Rev. A 1994 50, 5233-5241.

122. Lo Franco, R.; Compagno, G.; Messina, A.; Napoli, A. Generating and revealing a quantum superposition of electromagnetic-field binomial states in a cavity. Phys. Rev. A 2007, 76, 011804(R).

123. Lo Franco, R.; Compagno, G.; Messina, A.; Napoli, A. Generation of entangled two-photon binomial states in two spatially separate cavities. Open Syst. Inf. Dyn. 2006, 13, 463-470.

124. Lo Franco, R.; Compagno, G.; Messina, A.; Napoli, A. Single-shot generation and detection of a two-photon generalized binomial state in a cavity. Phys. Rev. A 2006, 74, 045803.

125. Lo Franco, R.; Compagno, G.; Messina, A.; Napoli, A. Bell's inequality violation for entangled generalized Bernoulli states in two spatially separate cavities. Phys. Rev. A 2005, 72, 053806.

126. Nußann, S.; Hijlkema, M.; Weber, B.; Rohde, F.; Rempe, G.; Kuhn, A. Submicron positioning of single atoms in a microcavity. Phys. Rev. Lett. 2005, 95, 173602.

127. Fortier, K.M.; Kim, S.Y.; Gibbons, M.J.; Ahmadi, P.; Chapman, M.S. Deterministic loading of individual atoms to a high-finesse optical cavity. Phys. Rev. Lett. 2007, 98, 233601.

128. Colombe, Y.; Steinmetz, T.; Dubois, G.; Linke, F.; Hunger, D.; Reichel, J. Strong atom-field coupling for Bose-Einstein condensates in an optical cavity on a chip. Nature 2007, 450, 272-276.

(c) 2015 by the authors; licensee MDPI, Basel, Switzerland. This article is an open access article distributed under the terms and conditions of the Creative Commons Attribution license (http://creativecommons.org/licenses/by/4.0/). 